\title{
Issues in the design of switched linear control systems: A benchmark study
}

\author{
Douglas Leith, Robert Shorten, William Leithead, Oliver Mason \\ The Hamilton Institute \\ NUI Maynooth \\ Ireland \\ Paul Curran \\ Department of Electronic and Electrical Engineering \\ NUI Dublin \\ Ireland
}

\begin{abstract}
In this paper we present a tutorial overview of some of the issues that arise in the design of switched linear control systems. Particular emphasis is given to issues relating to stability and control system realisation. A benchmark regulation problem is then presented. This problem is most naturally solved by means of a switched control design. The challenge to the community is to design a control system that meets the required performance specifications and permits the application of rigorous analysis techniques. A simple design solution is presented and the limitations of currently available analysis techniques are illustrated with reference to this example.
\end{abstract}

\section{Introductory remarks}

Recent years have witnessed an enormous growth of interest in dynamic systems that are characterised by a mixture of both continuous and discrete dynamics. Such systems are commonly found in engineering practice and are referred to as hybrid or switching systems. The widespread application of such systems is motivated by ever increasing performance requirements, and by the fact that high performance control systems can be realised by switching between relatively simple LTI systems. However, the potential gain of switched systems is offset by the fact that the switching action introduces behaviour in the overall system that is not present in any of the composite subsystems. For example, it can be easily shown that switching between stable sub-systems may lead to instability or chaotic behaviour of the overall system, or that switching between unstable sub-systems may result in a stable overall system.

In this paper we present a tutorial introduction to the design of switched linear systems. We begin by discussing how switching arises naturally in many situations. Examples include: the design of control systems for plants that are themselves characterised by switching action (i.e. plants with gears); the design of reconfigurable (fault tolerant) control systems; a switched controller that combines the advantages of several LTI controllers; and using switching to improve the transient response of adaptive control systems. We then discuss the issues in the design of such systems. Of primary practical importance are the issues of asymptotic stability, and issues concerning the realisation of switched linear controllers (and the associated transient response). Each of these issues is illustrated by means of simple illustrative examples.

The final part of the paper presents a wind turbine regulation problem. This problem is can be solved using a switched linear controller; the challenge to the community is to design such a controller, while providing theoretical guarantees concerning the issues raised in the paper. A control design is presented which is characterised by a number of switches, and exhibits control performance that is superior to single LTI and non-linear control design. Unfortunately, while this control system works well, both in simulation, and in practice, no guarantees can be given regarding performance and stability. In particular, the benchmark solution provides a basis for evaluating analysis techniques for 
analysing the stability of switched systems. In this context, a fundamental contribution of this paper is to document the limitations of these techniques and to motivate the study of theoretical issues that arise in the context of real industrial examples.

\section{The Need for Switching}

Switching between a number of control strategies has long been a valuable tool in the design of automatic control systems.

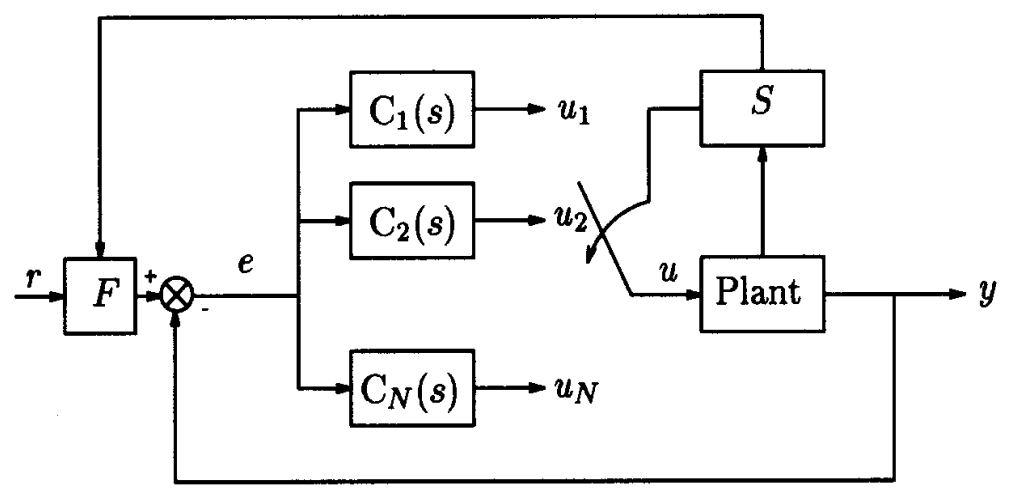

Figure 1: Schematic of a switching system. ' $S$ ' denotes a supervisory algorithm that controls the switching between the various controllers.

The need for (supervisory) switching arises for many reasons, some of which are listed below (Narendra et al., 1996, Goodwin, 2001):

(i) Plant dynamics: Many physical systems can be represented by switching or interpolating between locally valid models. Controllers that encompass switching are often a natural method for dealing with such systems.

(ii) Performance: Switching between a number of control structures automatically results in control systems that are no longer constrained by the limitations of linear design. It is therefore not surprising that switching based control strategies can result in algorithms that offer significant performance improvements over traditional linear control. For example, different controllers may be encoded within a single structure, resulting in a control system with enhanced functionality by exploiting the benefits of each of the constituent controllers.

(iii) Robustness: An important motivation for designing switching control strategies is to ensure robust control performance in the presence of component failure. For example, if an operating condition changes (a sensor failure, a change in sampling rate, or even a controller failure), then a more appropriate control action may be initiated by the supervisor. In extreme cases, switching to a new controller, or even continuous switching between a number of controllers, may be required to maintain closed loop stability.

(iv) Adaptive control: Much of the recent interest in switching systems has been motivated by developments in supervisory adaptive control. It is generally accepted that multiple-models, and multiple controllers are required in reconfigurable systems to detect and respond to changes in plant parameters and structure. The paradigm, multiple-models, switching and tuning, is based upon this assumption; namely, that by switching between several adaptive controllers, each initialised to different states, a rapidly converging adaptive control system may be constructed that is capable of coping with both unknown and time-varying parametric uncertainty (Narendra and Balakrishnan, 1994b, Narendra and Chen, 2002). 
(v) Decentralised design: Many complex engineering systems are designed in a decentralised manner. Each component sub-system is usually designed in relative isolation, and the overall system is constructed by combining each of the sub-systems by means of some appropriate supervisory logic. Often, this approach leads to switched linear control systems.

(vi) Control system constraints: Constraints are a common feature of practical control systems. Switching between several controllers is often a natural way of satisfying such constraints. Examples in this context are given in (Goodwin, 2002).

Despite the prevalence of switching based control algorithms in engineering design, such systems have only recently, in the context of the more general hybrid systems, attracted the interest of the academic community. Typically, switched linear systems are modelled by vector differential equations of the following form,

$$
\dot{\mathbf{x}}=\mathbf{A}(\mathbf{t}) \mathbf{x}+\mathbf{B}(\mathbf{t}) \mathbf{u}, \quad \mathbf{x}\left(\mathbf{t}_{0}\right)=\mathbf{x}_{0},
$$

where $\mathbf{x}(\mathbf{t}), \mathbf{u}(\mathbf{t}) \in \mathbf{R}^{\mathbf{n}}$ and where matrices $\mathbf{A}(\mathbf{t}), \mathbf{B}(\mathbf{t})$, are constructed by switching between a set of matrices. Formally we define a switched linear system, referred in the sequel to as the switching system, and its fundamental properties as follows (Shorten et. al., 2002).

The switching system: Consider the time-varying system

$$
\Omega: \dot{\mathbf{x}}(\mathbf{t})=\mathbf{A}(\mathbf{t}) \mathbf{x}+\mathbf{B}(\mathbf{t}) \mathbf{u}(\mathbf{t}) \quad, \quad \mathbf{x}\left(\mathbf{t}_{\mathbf{0}}\right)=\mathbf{x}_{\mathbf{0}}
$$

where $\mathbf{x}(\mathbf{t}) \in \mathrm{R}^{\mathrm{n}}, \mathbf{u}(\mathbf{t}) \in \mathrm{R}^{\mathrm{p}}$ and where matrices $\mathbf{A}(\mathbf{t}), \mathbf{B}(\mathbf{t})$, are constructed by switching between a set of stable matrices, $\mathbf{A}(\mathbf{t}) \in\left\{\mathbf{A}_{1}, \mathbf{A}_{2}, \ldots . ., \mathbf{A}_{\mathrm{m}}\right\}, \mathbf{B}_{\mathbf{i}} \in \mathbf{R}^{\mathrm{n} \times \mathbf{n}}, \mathbf{B}(\mathbf{t}) \in\left\{\mathbf{B}_{1}, \mathbf{B}_{2}, \ldots ., \mathbf{B}_{\mathrm{m}}\right\}, \mathbf{B}_{\mathbf{i}} \in \mathbf{R}^{\mathrm{n} \times \mathbf{p}}$ and where for each time $t$ the matrices $A(t), B(t)$ equals one and only one matrix $\mathbf{A}_{\mathbf{i}}, \mathbf{B}_{\mathbf{i}}$ in the above sets. Typically the matrix pairs $\left(\mathbf{A}_{\mathbf{i}}, \mathbf{B}_{\mathbf{i}}\right)$ are chosen such that equation (1) is bounded-input bounded-output stable for all fixed values of $t$ (Khalil, 1992). This corresponds to switching between a number of stable systems. Further, we assume that once the matrices $\mathbf{A}(\mathbf{t}), \mathbf{B}(\mathbf{t})$ assume the values $\mathbf{A}_{\mathbf{i}}, \mathbf{B}_{\mathbf{i}}$ they assume these values for an interval of time $\tau$ where $\tau \geq \tau_{\min }>0$, where the constant $\tau_{\min }$ is arbitrarily small and independent of $i$. For instance, suppose that the dynamics in (1) are given by $\dot{\mathbf{x}}=\mathbf{A}_{\mathrm{i}} \mathbf{x}+\mathbf{B}_{\mathrm{i}} \mathbf{u}$ over the finite time interval $\left[t_{\gamma}, t_{\gamma+1}\right)$. At time $t_{\gamma+1}$ the system switches and the dynamics in the following interval $\left[t_{\gamma+1}, t_{\gamma+2}\right)$ are given by $\dot{\mathbf{x}}=\mathbf{A}_{\mathbf{j}} \mathbf{x}+\mathbf{B}_{\mathbf{j}} \mathbf{u}$. We assume that the state vector $x(t)$ does not jump discontinuously at $t_{\gamma+1}$, and hence the initial state at time $t_{\gamma+1}$ for $\dot{\mathbf{x}}=\mathbf{A}_{\mathbf{j}} \mathbf{x}+\mathbf{B}_{\mathbf{j}} \mathbf{u}$ is the terminal state of $\dot{\mathbf{x}}=\mathbf{A}_{\mathbf{i}} \mathbf{x}+\mathbf{B}_{\mathbf{i}} \mathbf{u}$. If we further assume that $\mathbf{u}=\mathbf{K}_{\mathbf{i}} \mathbf{x}$ then the following convenient representation of (1) is obtained,

$$
\Omega: \dot{\mathbf{x}}=\overline{\mathbf{A}}(\mathbf{t}) \mathbf{x} \quad \mathbf{x}\left(\mathbf{t}_{0}\right)=\mathbf{x}_{0}
$$

where $\overline{\mathbf{A}}(\mathbf{t}) \in\left\{\overline{\mathbf{A}}_{1}, \overline{\mathbf{A}}_{2}, \ldots ., \overline{\mathbf{A}}_{\mathbf{m}}\right\}, \quad$ and $\overline{\mathbf{A}}_{\mathbf{i}}=\mathbf{A}_{\mathbf{i}}+\mathbf{K}_{\mathbf{i}} \mathbf{B}_{\mathbf{i}}$. We refer to systems (1) and (2) interchangeably as the switching system.

Associated with the switching system (1) we also define the $i^{\text {th }}$ constituent system, the switching sequence $S W$ and a switching signal $\rho(t)$.

The $i^{\text {th }}$ constituent system: Consider the linear time-invariant system

$$
\Sigma_{\mathrm{i}}: \dot{\mathbf{x}}=\mathbf{A}_{\mathrm{i}} \mathbf{x}+\mathbf{B}_{\mathrm{i}} \mathbf{u}
$$

with the switching system defined above. Then $\Sigma_{i}$ is referred to as the $i^{\text {th }}$ constituent system of (1). 
The switching sequence $S W$ : In the spirit of (Branicky, 1994) one can associate the following switching sequence with (1)

$$
S W=\left(i_{0}, t_{0}\right),\left(i_{1}, t_{1}\right), \ldots . .,\left(i_{N}, t_{N}\right), \ldots \ldots
$$

where the $S W$ sequence may or may not be infinite. The $\gamma^{\text {th }}$ switching interval is the $\gamma^{\text {th }}$ element of this sequence and defines the evolution of (1) as follows. The switching system evolves according to $\Sigma_{\mathrm{i}_{\gamma}}: \dot{\mathrm{x}}=\mathbf{A}_{\mathrm{i} \gamma} \mathbf{x}+\mathbf{B}_{\mathrm{i}_{\gamma}} \mathbf{u}$ for $t_{\gamma} \leq t<t_{\gamma+1}$, with the initial condition given by $\mathbf{x}\left(\mathrm{t}_{\gamma}\right)$.

The switching signal: Let $\rho(t): R \rightarrow R$ be a piecewise constant function, $\rho(t) \in\{1, \ldots, m\}$ for all $t$. Suppose that the switching sequence $S W$ is chosen such that $\mathbf{A}(\mathbf{t})=\mathbf{A}_{\rho(\mathbf{t})}$ for all t. Then, $\rho(t)$ is said to be a switching signal for the system (1).

\section{Issues in the Design of Switching Systems}

Equations of the form of (1) have been the subject of much attention in the Mathematics, and more recently in the Systems Science community. However, despite much effort, relatively little is know about the qualitative properties of their solutions. From a practical viewpoint, the design of switching systems is characterised by a number of specific issues that ultimately determine the applicability of a given control strategy. Issues that are of particular interest to engineers are stability problems associated with switched linear systems, the transient response properties of such systems, and the associated issue of controller realisation. These issues have now become the focus of recent work in this area.

\subsection{Stability problems associated with switching systems}

It is well known a switching system can be potentially destabilised by an appropriate choice of switching signal, even if the switching is between a number of Hurwitz-stable closed loops systems. Even in the case where the switching is between systems with identical transfer functions, it is sometimes possible to destabilise the switching sytem by means of switching.

Example 1: Consider the time-varying system

$$
\begin{aligned}
\Omega: & \dot{\mathbf{x}}=\mathbf{A}(\mathbf{t}) \mathbf{x}+\mathbf{B}^{\mathrm{T}} \mathbf{C} \mathbf{u}, \\
& \mathbf{u}=\mathbf{x},
\end{aligned}
$$

where $\mathbf{x} \in \mathbf{R}^{2}, \mathbf{A}(\mathbf{t}) \in\left\{\mathbf{A}_{1}, \mathbf{A}_{2}\right\}, \mathbf{B}(\mathbf{t}) \in\left\{\mathbf{b}_{1}, \mathbf{b}_{2}\right\}$ and $\mathbf{C}(\mathbf{t}) \in\left\{\mathbf{c}_{1}, \mathbf{c}_{2}\right\}$ with

$$
\mathbf{A}_{1}=\left[\begin{array}{cc}
0 & 1 \\
-3 & -0.2
\end{array}\right], \quad \mathbf{A}_{2}=\left[\begin{array}{cc}
-0.2 & -3 \\
1 & 0
\end{array}\right],
$$

and $\boldsymbol{b}_{1}=\left[\begin{array}{ll}0 & 1\end{array}\right], \boldsymbol{c}_{1}=\left[\begin{array}{ll}1 & 0\end{array}\right], \boldsymbol{b}_{2}=\left[\begin{array}{ll}1 & 0\end{array}\right], \boldsymbol{c}_{2}=\left[\begin{array}{ll}0 & 1\end{array}\right]$. The system $\Omega$ can be thought of as being constructed by switching between the vector fields associated with the linear time invariant (LTI) systems,

$$
\begin{aligned}
\Sigma_{\mathrm{i}}: \quad \dot{\mathbf{x}} & =\mathbf{A}_{\mathrm{i}} \mathbf{x}+\mathbf{B}_{\mathrm{i}}^{\mathrm{T}} \mathbf{C}_{\mathrm{i}} \mathbf{u}, \\
\mathbf{u} & =\mathbf{x},
\end{aligned}
$$


with the $\left(\mathbf{A}_{\mathbf{i}}, \mathbf{B}_{\mathbf{i}}, \mathbf{C}_{\mathbf{i}}\right)$ defined above. The system matrices are given by $\left\{\mathbf{A}_{1}+\mathbf{B}_{1}^{\mathrm{T}} \mathbf{C}_{\mathbf{i}}, \mathbf{A}_{2}+\mathbf{B}_{2}^{\mathrm{T}} \mathbf{C}_{2}\right\}$. Here, each of the closed loop matrices to have identical eigenvalues, lying in the left-half of the complex plane (Hurwitz-stable); hence the transfer functions $\mathbf{C}_{\mathbf{i}}\left(\mathbf{s I}-\mathbf{A}_{\mathbf{i}}\right)^{-1} \mathbf{B}_{\mathbf{i}}^{\mathrm{T}}$ are identical. The vector field associated with each of the closed loop system matrices is depicted in Figure 3.

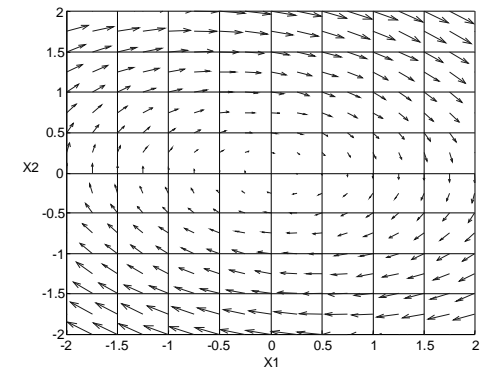

(a) The vector field $\Sigma_{1}$.

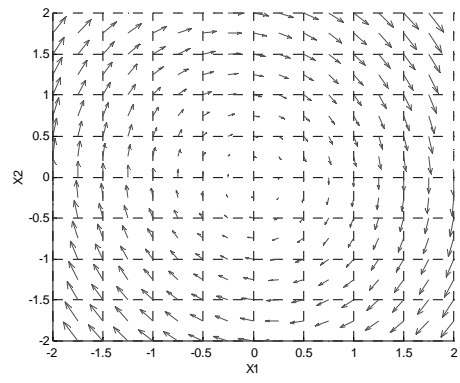

(b) The vector field $\Sigma_{2}$.

Figure 3: The vector fields $\Sigma_{1}$ and $\Sigma_{2}$.

Clearly, the switching system is constructed by switching between two stable vector fields. However, the solution to the system $\Omega$, with initial condition $\mathbf{x}_{0}^{\mathbf{T}}=[10]$, which can be written, $\mathbf{x}_{0}^{\mathbf{T}}=[10]$,

$$
M(t)=e^{Z_{1} t_{1}} e^{Z_{2} t_{2}} \cdots e^{Z_{N} t_{n}}
$$

at time $t=\sum_{i=1}^{n} t_{i}$ is unbounded for a periodic switching sequence with $\mathbf{Z}_{2 \mathrm{i}}=\mathbf{A}_{1}, \mathbf{Z}_{2 \mathrm{i}+1}=\mathbf{A}_{2}$, $i=\{0, \ldots, m\}$, and $t_{i}=0.5$. This is depicted in Figure 4 .

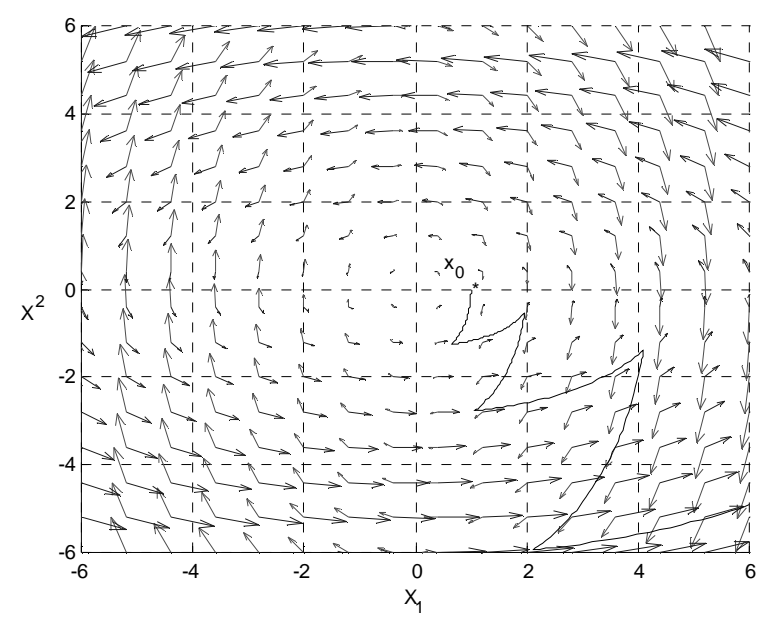

Figure 4

It is clear from the above example that instability may arise in switched linear systems, even if the switching occurs between systems that are themselves exponentially stable. Instability arises in such systems due to the fact that the instability mechanism depends not only on the eigenvalues but also upon the eigenvectors of the constituent matrices, as well as the choice of switching signal $\rho(t)$. Clearly, depending upon application, the control system designer may be able, by means of control design,to influence some or all of the above factors. In this context a number of stability problems arise naturally when discussing switching systems. In the following discussion we use the term 
stability in an abstract sense, meaning where appropriate, uniform (with respect to initial condition) global exponential or asymptotic stability.

\section{(i) Arbitrary switching}

In many engineering problems, restrictions on the switching signal cannot be specified a-priori. For such applications, the problem of stability is to obtain verifiable conditions on the matrices $\left(A_{i}, B_{i}\right)$ that guarantee the asymptotic stability of the switching system (1) for any switching signal $\rho(t)$. Clearly, for this problem to be solvable the system must be asymptotically stable for constant switching sequences, and thus each of the constituent systems must be asymptotically stable. Much of the recent work on the stability of switched linear systems has focussed on this problem; see (Curran 98, Shorten and Narendra, 1998b, Liberzon and Morse, 1999; DeCarlo et al, 2000; Shorten er al., 2002b) for an overview of work completed in this area. Typically, the approach taken here is to develop design laws that guarantee the existence of a Lyapunov function, not necessarily quadratic, that is common to all of the constituent systems. Consequently, much of the work carried out in this area has focussed on developing existence laws (or algorithms) for certain types of Lyapunov function (Narendra and Balakrishnan, 1994a; Mori et al. 1997, Liberzon et. al. 1998; Shorten and Narendra, 1998a, Shorten and Narendra, ).

\section{(ii) The dwell-time problem}

Even if the switching system (1) fails to be stable for all possible switching sequences, there may be many practically useful sequences for which it is asymptotically stable. The dwell-time problem is to explicitly determine a minimum time between switches, $\tau_{\min }$, such that stability is maintained. More generally we are interested in determining all switching signals that result in instability for the system.

Example 2: Consider the time-varying system given by the following scalar differential equation

$$
\ddot{y}+0.2 \dot{y}+(1-0.5 \mathrm{~g}(\mathrm{t})) \mathrm{y}=0,(4)
$$

where $y(t)$ is a scalar function of $t$, and $g(t)$ is some scalar periodic signal that takes the value -1 or 1 . This system can be represented in state space form,

$$
\Omega: \quad \dot{\mathbf{x}}=\mathbf{A}(\mathbf{t}) \mathbf{x}
$$

where $\mathbf{x}=[\mathbf{y}, \dot{\mathbf{y}}], \mathbf{A}(\mathbf{t}) \in\left\{\mathbf{A}_{1}, \mathbf{A}_{2}\right\}$, with

$$
\mathbf{A}_{1}=\left[\begin{array}{cc}
0 & 1 \\
-0.5 & -0.2
\end{array}\right], \quad \mathbf{A}_{2}=\left[\begin{array}{cc}
0 & 1 \\
-1.5 & -0.2
\end{array}\right],
$$

As in example (1), the system $\Omega$ can be thought of as being constructed by switching between the vector fields associated with the linear time invariant (LTI) systems,

$$
\Sigma_{\mathrm{i}}: \quad \dot{\mathbf{x}}=\mathbf{A}_{\mathrm{i}} \mathbf{x}
$$

with the $\mathbf{A}_{\mathbf{i}}$ defined above. The vector fields associated with each constituent system are depicted in Figure 5. 


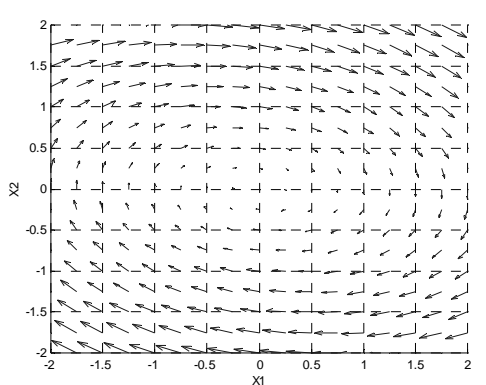

(a) The vector field $\Sigma_{1}$.

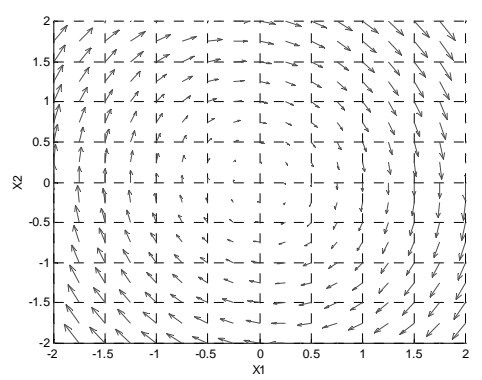

(b) The vector field $\Sigma_{1}$.

Figure 5: The vector fields $\Sigma_{1}$ and $\Sigma_{2}$.

In this example, the form of Equation (4) admits approximate analysis along the lines of describing function techniques presented in (Khalil, 92)

$$
\begin{aligned}
& \ddot{y}+0.2 \ddot{y}+f(t) y=0 \\
& \Rightarrow \ddot{y}+0.2 \ddot{y}+\left(f_{0}+f_{1} \cos \left(\omega_{0} t+\phi\right)+\text { h.o.t. }\right) y=0
\end{aligned}
$$

where $\omega_{0}=\frac{2 \pi}{T}, T$ is the period of $f(t)$, and where $f_{0}+f_{1} \cos \left(\omega_{0} t+\varphi\right)+$ h.o.t. is the Fourier series representation of $f(t)$. If we assume that the system has a low-pass characteristic, then we may approximate the system $\mathrm{f}(\mathrm{t}) \approx \mathrm{f}_{0}+\mathrm{f}_{1} \cos (\omega \mathrm{t}+\phi)$. Hence,

$$
\begin{aligned}
& \Rightarrow \ddot{y}+0.2 \ddot{y}+\left(f_{0}+f_{1} \cos (\omega t+\phi)\right) y=0 \\
& \Rightarrow Y(j \omega)=\left\|\frac{-f_{1}}{2\left(s^{2}+0.2 s+f_{0}\right)}\right\|_{s=j \omega}\left(Y \left(j\left(\omega-\omega_{0}-\phi-\theta(\omega)\right)+Y\left(j\left(\omega+\omega_{0}+\phi+-\theta(\omega)\right)\right)\right.\right.
\end{aligned}
$$

Now consider the existence of a solution of the form $\cos \left(\frac{\omega_{0}}{2} t+\frac{\varphi}{2}+\frac{\theta\left(\omega_{0}\right)}{2}\right)$ where $\theta\left(\omega_{0}\right)$ is the phase response of the transfer function at $\omega_{0}$. If we again assume a low-pass system characteristic, the equation (5) yields:

$$
\cos \left(\frac{\omega_{0}}{2} t+\frac{\varphi}{2}+\frac{\theta\left(\omega_{0}\right)}{2}\right)=\left\|\frac{-f_{1}}{2\left(s^{2}+0.2 s+f_{0}\right)}\right\|_{s=j \omega} \cos \left(\frac{\omega_{0}}{2} t+\frac{\varphi}{2}+\frac{\theta\left(\omega_{0}\right)}{2}\right)
$$

Clearly, the above equation predicts instability everywhere the magnitude of Bode plot of the transfer function

$$
H(s)=\frac{-f_{1}}{2\left(s^{2}+0.2 s+f_{0}\right)}
$$

exceeds unity. This Bode plot for a given periodic $\mathrm{f}(\mathrm{t})$ is given Figure 6. 

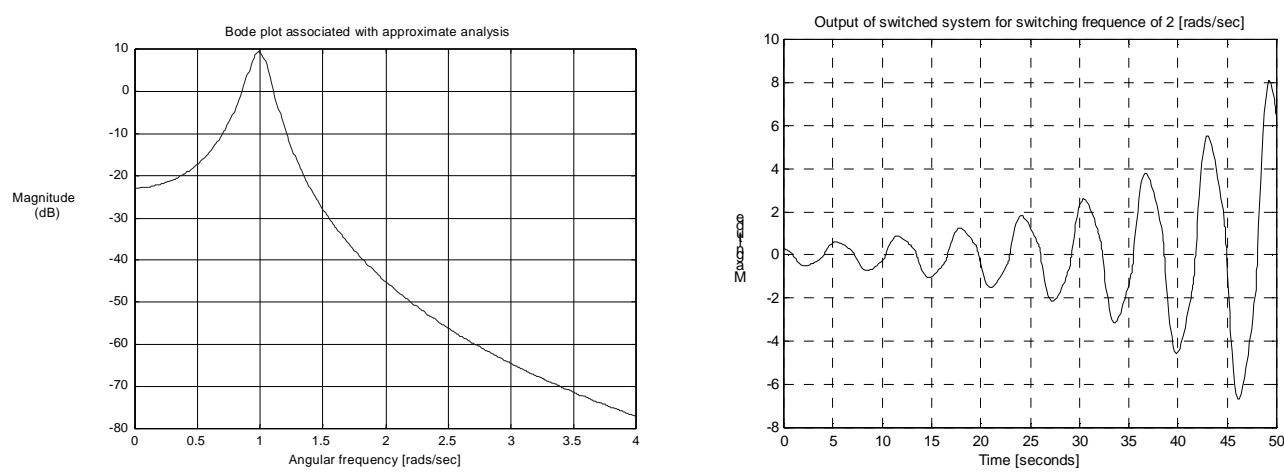

Figure 6: Response of system

Clearly, instability is predicted for a range of switching frequencies.

The above example is extremely interesting as it indicated the existence of a band of unstable switching sequences, sandwiched between, bands of stable switching sequences. While the above analysis technique provides a reliable method for the determination of this band of frequencies, techniques for analysing general MIMO switching systems are not available. Note also that the instability mechanism in Example 2 differs qualitatively from the instability mechanism in Example 1. In Example 1, instability is induced via unstable chattering, whereas in Example 2 instability is induced via spirals of increasing amplitude. Qualitatively speaking, the system in Example 1 becomes more unstable the faster one switches, whereas the system in Example 2 is unstable for a selective set of switching signals.

\section{(iii) Routes to instability}

Examples (1) and (2) illustrate two possible instability mechanisms in switching systems. An open question in the study of switching systems concerns the possible routes to instability. While early work by (Pyatnitski and Rappaport, 1992) and his co-workers on SISO systems suggests that the boundary of stability may be characterised by the existence of marginally stable periodic switching signal, more recent work (Blondel, 2000) suggests that this is, in general, not true. It is therefore of interest to identify particular instability mechanisms for a given switching system. Examples of instability mechanisms include: periodic chattering (Example 1); and periodic spiraling (Example 2). Knowledge of the instability mechanism for a class of system allows one to design non-conservative stability criteria. Hence, problem (iii) is to describe and classify these mechanisms. Initial results in this direction, and in the derivation of non-conservative stability criteria, are reported in (Shorten et al. 2000b, Wulff et. al, 2002).

\section{(iv) Robustness of stability criteria}

The concept of robustness with respect to parameter variations is well defined for LTI systems. This issue is somewhat more difficult to quantify for switched linear systems. In particular, robustness may be defined with respect to a number of design parameters, including, not only the parameters of the closed loop system matrices, but also with respect to switching signal $\rho(t)$.

\section{Example 3:}

Consider the following system

$$
\dot{\mathbf{x}}=\mathbf{A}(\mathbf{t}) \mathbf{x}, \mathbf{x}\left(\mathbf{t}_{0}\right)=\mathbf{x}_{0},
$$


where $\mathbf{x}(\mathbf{t}) \in \mathbf{R}^{\mathrm{n}}$ and where matrices $A(t)$ is constructed by switching between a set of matrices; $\mathbf{A}(\mathbf{t}) \in\left\{\mathbf{A}_{1}, \mathbf{A}_{2}\right\}, \mathbf{A}_{\mathbf{i}} \in \mathbf{R}^{2 \times 2}$, and where

$$
\mathbf{A}_{1}=\left[\begin{array}{cc}
-\mathrm{K} & 0 \\
0 & -\frac{1}{\mathrm{~L}^{2}}
\end{array}\right], \mathbf{A}_{2}=\mathbf{M}\left[\begin{array}{cc}
-\mathrm{K} & 0 \\
0 & -\frac{1}{\mathrm{~L}^{2}}
\end{array}\right] \mathbf{M}^{-1}, \mathbf{M}=\left[\begin{array}{cc}
1 & \frac{1}{\mathrm{~L}} \\
\frac{1}{\mathrm{~L}} & 1
\end{array}\right],
$$

where $K, L>0$. The existence of a common quadratic Lyapunov function for the constituent systems $\Sigma_{1}$ and $\Sigma_{2}$ is a sufficient condition for asymptotic stability of the switching system for arbitrary $\rho(t)$. A sufficient condition for the existence of such a function is that the matrices $\mathbf{A}_{1}$ and $\mathbf{A}_{2}$ are Hurwitz, and that a linear transformation $\mathbf{T}$ exists such that $\mathbf{T} \mathbf{A}_{\mathbf{1}} \mathbf{T}^{-\mathbf{1}}$ and $\mathbf{T} \mathbf{A}_{2} \mathbf{T}^{-1}$ are upper triangular matrices (Mori et. al, 1997, Shorten and Narendra 1998a).

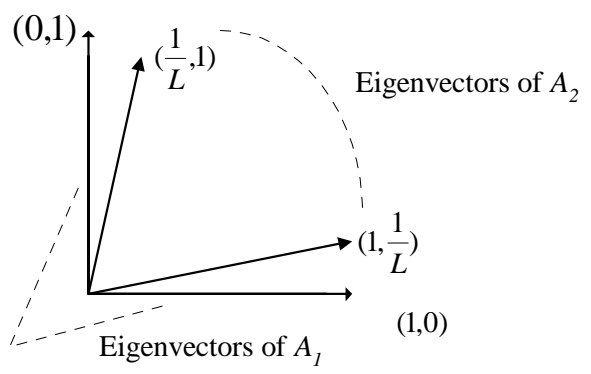

Clearly, the matrices $A_{1}, A_{2}$ and $M$ the following properties:

(a) $\mathbf{A}_{1}$ and $\mathbf{A}_{2}$ have identical eigenvalues.

(b) $\lim _{\mathrm{L} \rightarrow \infty} \mathbf{A}_{1}(\mathrm{~L})=\lim _{\mathrm{L} \rightarrow \infty} \mathbf{A}_{2}$ (L)

(c) $\lim _{\mathrm{L} \rightarrow \infty} \mathbf{M}(\mathrm{L})=\mathrm{I}$

Hence, the matrices $\mathbf{A}_{\mathbf{1}}, \mathbf{A}_{\mathbf{2}}$, can be made arbitrarily close to one another, while at the same time remaining Hurwitz-stable. In view of the above properties it would not be unreasonable to expect that the above switching system is asymptotically stable for $L$ large enough. However, it is shown in (Shorten et. al, 2000) that given any $L>1$, there always exists a positive $K$ such that an unstable switching sequence exists for the switching system. Hence, while the existence of a quadratic Lyapunov function for an LTI system provived a quantifiable degree of robustness, arbitrarily small perturbations on the parameters of a switching system can destroy not only the existence of such a function, but also the stability of the underlying switching system.

\section{(v) Numerical approaches to stability: A cautionary word}

As mentioned earlier, the existence of a common quadratic Lyapunov function for each of the constituent systems of a switched linear system is sufficient to guarantee the asymptotic stability of the overall system for arbitrary switching signals. The problem of determining whether or not such a function exists can readily be formulated as a feasibility problem for a system of linear matrix inequalities or LMIs (Boyd et al, 1994). Recent increases in computational power together with the development of efficient numerical algorithms (in the field of convex optimisation) for solving LMIrelated problems has led to a considerable increase in popularity for this method of establishing the stability of switched systems. Furthermore, for certain subclasses of switched linear systems, such as piecewise linear systems where switching is determined by the values of the state variables, other types of Lyapunov function can be used to establish asymptotic stability. For example, the asymptotic stability of piecewise linear systems can be established using piecewise quadratic Lyapunov functions (Johansson and Rantzer, 1998), (Pettersson and Lennartson, 1997), and the search for such functions can again be formulated as a system of LMIs which can be solved using the MATLAB LMI toolbox or 
any of a number of available software packages. We shall discuss the application of some these techniques to the benchmark example in section 5.2.

\section{Remark}

While the numerical methods based on LMIs can be used effectively to establish the stability of a large class of systems, it is important to point out some drawbacks of the approach. First of all, the system parameters must be estimated or known before performing the numerical test for stability. Thus, while LMIs can be used to test a system for stability, they do not provide usable design laws that guarantee stability.

More significantly, the numerical approach can fail to give the correct answer in some cases even for classes of systems for which theoretical results are well established. For an example of this phenomenon, see the stability analysis of the benchmark example in section 5.2.

To further illustrate the point, we present the following example.

\section{Example 4:}

Consider the following system

$$
\dot{\mathbf{x}}=\mathbf{A}(\mathbf{t}) \mathbf{x}, \mathbf{x}\left(\mathbf{t}_{0}\right)=\mathbf{x}_{0},
$$

where $\mathbf{x}(\mathbf{t}) \in \mathbf{R}^{\mathrm{n}}$ and where matrices $\mathbf{A}(\mathbf{t})$ are constructed by switching between a set of matrices;

$\mathbf{A}(\mathbf{t}) \in\left\{\mathbf{A}_{1}, \mathbf{A}_{2}\right\}, \mathbf{A}_{\mathbf{i}} \in \mathbf{R}^{2 \times 2}$, with

$$
A_{1}=\left[\begin{array}{ll}
-1 & 1 \\
0 & -10^{-20}
\end{array}\right] \quad A_{2}=\left[\begin{array}{ll}
-1 & 1.0000001 \\
0 & -10^{-20}
\end{array}\right]
$$

Now it is well known that when the system matrices of the constituent systems are Hurwitz-stable and upper triangular, the switching system possesses a common quadratic Lyapunov function. However, in this case the MATLAB LMI toolbox fails to find a common quadratic Lyapunov function for the system. (NOTE: This example is of course contrived and is included primarily to illustrate the above point. For a more realistic example see the analysis in section 5.2.)

\subsection{Controller realisation and transient response}

The issue of transient response is perhaps, from the viewpoint of practical design, as important as the issue of closed loop stability. Clearly, the presence of a switching element as part of a feedback loop is likely to cause transients. Roughly speaking, the transient response properties of the feedback loop are determined by the eigenvectors, and the eigenvalues, of the closed loop feedback matrices ( $A_{i}$ and $\bar{A}_{i}$ in Equations (1) and (2) respectively). A comprehensive discussion of shaping the transient response properties of a switched linear system is beyond the scope of this paper; see (Shorten \& Narendra, $1998 b$ ) for a discussion of the issues involved. Fortunately, several degrees of freedom exist that allow us to manipulate the transient properties of the closed loop system. Here we illustrate, by means of a simple example, several degrees of freedom in the controller realisation that can be manipulated to influence not only the stability properties of the closed loop, but also the transient response properties. In particular, we consider whether the control system should be implemented as a global state controller, or as a local state controller, or as a mixture of both global and local states. The important related issues of state initialisation and the position of the switches in the feedback loop are not discussed due to space limitations. 
Example 4: Consider the following first order time-varying plant

$$
\dot{y}+a_{i} y=K_{p i} u,
$$

where $y$ and $u$ are scalars and where $a_{i} \in\left\{a_{1}, a_{2}\right\}, K_{p i} \in\left\{K_{p 1}, K_{p 2}\right\}$. Further, we make the unrealistic assumption that the exact instant at which the plant parameters switch is known, and that this information can be instantaneously utilised to switch the controller parameters. We adopt a pole placement design, and our design objective is to place the poles of the closed loop system at fixed locations (independent of switching signal), $\lambda_{1}, \lambda_{2}, \lambda_{3}$, while maintaining unity gain between the command input and output. We consider a controller structure of the following form,

$$
C(s)=\frac{1}{s} \frac{K_{1 i} s+K_{2 i}}{\left(s+b_{i}\right)}
$$

and we investigate three implementations of this control structure; a global state approach; a local state approach; and a factored local state approach (both global and local states). In each case we assume a constant input of unity.

(i) Global state controller

In this case the control system can be written,

$$
\begin{gathered}
\ddot{u}+b_{i} \dot{u}=K_{1 i} \dot{e}+K_{2 i} e \\
e=r-y \\
r=G_{i} u_{c}
\end{gathered}
$$

where $u_{c}$ is the scalar command signal, and where $b_{i} \in R^{+}, K_{i 1}, K_{i 0}, G \in R$. . By choosing the state vector as $\mathbf{x}=[\dot{\mathrm{u}}, \mathrm{u}, \mathrm{y}]^{\mathrm{T}}$, the following state space representation of the closed loop system is obtained,

$$
\dot{\mathbf{x}}=\mathbf{A}(\mathbf{t}) \mathbf{x}+\mathbf{B}(\mathbf{t}) \mathbf{r}
$$

where $r=\left[u_{c}\right], \mathbf{A}(\mathbf{t}) \in\left\{\mathbf{A}_{1}, \mathbf{A}_{2}\right\}, \mathbf{B}(\mathbf{t}) \in\left\{\mathbf{B}_{1}, \mathbf{B}_{2}\right\}$, and where

$$
\mathbf{A}_{\mathrm{i}}=\left[\begin{array}{ccc}
-\mathrm{b}_{\mathrm{i}} & \mathrm{K}_{\mathrm{pi}} \mathrm{K}_{1 \mathrm{i}} & \mathrm{K}_{\mathrm{li}} \mathrm{a}_{\mathrm{i}}-\mathrm{K}_{2 \mathrm{i}} \\
1 & 0 & 0 \\
0 & \mathrm{~K}_{\mathrm{pi}} & -\mathrm{a}_{\mathrm{i}}
\end{array}\right], \quad \mathbf{B}_{\mathrm{i}}=\left[\begin{array}{c}
\mathrm{K}_{21} \mathrm{G}_{\mathrm{i}} \\
0 \\
0
\end{array}\right]
$$

and $u_{c}=1$.

(ii) Local state controller

In this case the controllers take the form

$$
\begin{aligned}
& \ddot{u}_{1}+b_{1} \dot{u}_{1}=K_{11} \dot{e}+K_{21} e \\
& \ddot{u}_{2}+b_{2} \dot{u}_{2}=K_{12} \dot{e}+K_{22} e
\end{aligned}
$$


with $e$ defined above. A supervisor switches between the local state controller outputs according to $u(t)=\alpha(t) u_{1}+(1-\alpha(t)) u_{2}$ where the signal $\alpha(t)$ takes the value 0 when controller 2 is active, and the value 1 when controller 1 is active.

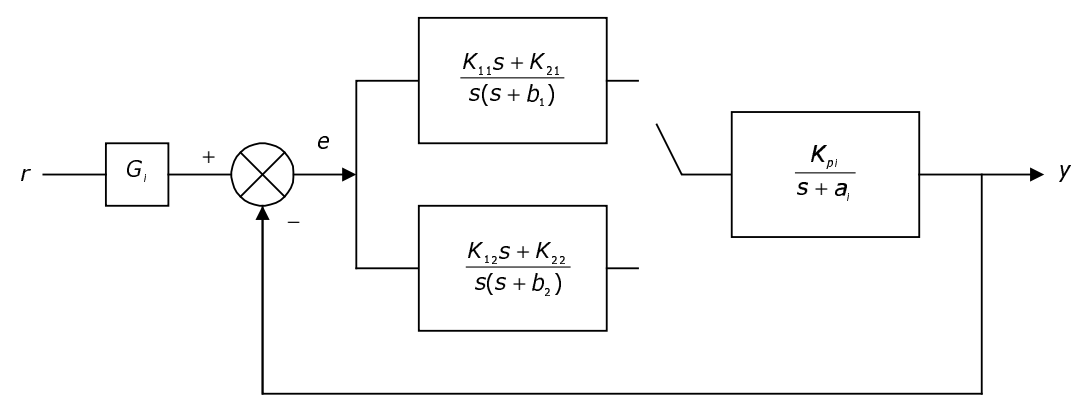

Figure 8: Schematic of local state controller for fixed value of $t$.

With the state vector defined $x=\left[u_{1}, \dot{u}_{1}, u_{2}, \dot{u}_{2}, y\right]^{T}$ the corresponding state space equation can be written,

$$
\dot{\mathbf{x}}=\mathbf{A}(\mathbf{t}) \mathbf{x}+\mathbf{B}(\mathbf{t}) \mathbf{r}
$$

where $r=\left[u_{c}\right], \mathbf{A}(\mathbf{t}) \in\left\{\mathbf{A}_{1}, \mathbf{A}_{2}\right\}, \mathbf{B}(\mathbf{t}) \in\left\{\mathbf{B}_{1}, \mathbf{B}_{2}\right\}$,

$$
\begin{gathered}
\mathbf{A}_{\mathbf{i}}=\left[\begin{array}{ccccc}
0 & 1 & 0 & 0 & 0 \\
\alpha(\mathrm{t}) \mathrm{K}_{11} \mathrm{~K}_{\mathrm{pi}} & -\mathrm{b}_{1} & -(1-\alpha(\mathrm{t})) \mathrm{K}_{11} \mathrm{~K}_{\mathrm{p} 2} & 0 & \mathrm{~K}_{11} \mathrm{a}_{\mathrm{i}}-\mathrm{K}_{21} \\
0 & 0 & 0 & 1 & 0 \\
\alpha(\mathrm{t}) \mathrm{K}_{21} \mathrm{~K}_{\mathrm{pi}} & 0 & -(1-\alpha(\mathrm{t})) \mathrm{K}_{21} \mathrm{~K}_{\mathrm{p} 2} & -\mathrm{b}_{2} & \mathrm{~K}_{12} \mathrm{a}_{\mathrm{i}}-\mathrm{K}_{22} \\
\alpha(\mathrm{t}) \mathrm{K}_{\mathrm{pi}} & 0 & (1-\alpha(\mathrm{t})) \mathrm{K}_{\mathrm{pi}} & 0 & -\mathrm{a}_{\mathrm{i}}
\end{array}\right], \\
\mathbf{B}_{\mathbf{i}}=\left[\begin{array}{c}
0 \\
\mathrm{~K}_{21} \mathrm{G}_{1} \\
0 \\
\mathrm{~K}_{21} \mathrm{G}_{1} \\
0
\end{array}\right] .
\end{gathered}
$$

(iii) Factored local state approach (global and local controller states):

Both controllers exhibit integral action. Hence, it is possible to factor the integrator out of both controllers and include it to, algebraically, as part of the plant. In this case the controllers and the augmented plant take the form

$$
\begin{aligned}
& \ddot{y}+a_{i} \dot{y}=K_{p i} u \\
& \dot{u}_{1}+b_{1} u_{1}=-K_{11} \dot{e}-K_{21} e \\
& \dot{u}_{2}+b_{2} u_{2}=-K_{12} \dot{e}-K_{22} e
\end{aligned}
$$

with $e$ defined as before. A supervisor switches between the local state controller outputs according to $u(t)=\alpha(t) u_{1}+(1-\alpha(t)) u_{2}$ as above. 


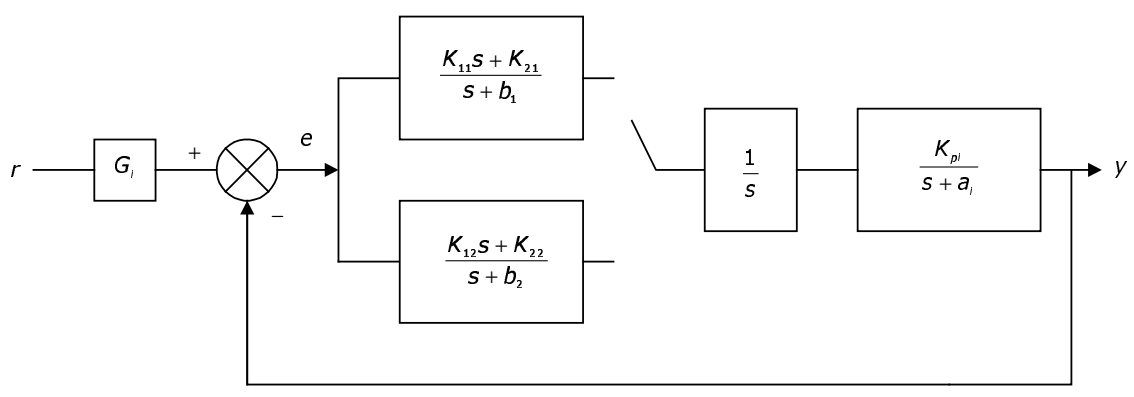

Figure 9: Schematic of factored implementation for fixed value of $t$.

With the state vector defined $x=\left[u_{1}, u_{2}, \dot{y}, y\right]^{T}$ the corresponding state space equation can be written,

$$
\mathbf{A}_{\mathrm{i}}=\left[\begin{array}{cccc}
-\mathrm{b}_{1} & 0 & -\mathrm{K}_{11} & -\mathrm{K}_{12} \\
0 & -\mathrm{b}_{2} & -\mathrm{K}_{12} & -\mathrm{K}_{22} \\
\alpha(\mathrm{t}) \mathrm{K}_{\mathrm{pi}} & (1-\alpha(\mathrm{t})) \mathrm{K}_{\mathrm{pi}} & -\mathrm{a}_{\mathrm{i}} & 0 \\
0 & 0 & 1 & 0
\end{array}\right], \quad \mathbf{B}_{\mathrm{i}}=\left[\begin{array}{c}
\mathrm{K}_{21} \mathrm{G} \\
\mathrm{K}_{21} \mathrm{G} \\
0 \\
0
\end{array}\right],
$$

We note that the parameters of the controllers are identical. Cases (i)-(iii) differ only in implementation. We present one comparative example with the closed loop poles located at [-0.2,$1.2,-5]$. The controller parameters for the example is given in Tables 1 .

\begin{tabular}{|c|c|c|c|c|c|c|}
\hline $\boldsymbol{i}$ & $\boldsymbol{a}_{\boldsymbol{i}}$ & $\boldsymbol{K}_{p \boldsymbol{i}}$ & $\boldsymbol{b}_{\boldsymbol{i}}$ & $\boldsymbol{K}_{\boldsymbol{1 i}}$ & $\boldsymbol{K}_{2 \boldsymbol{i}}$ & $\boldsymbol{G}_{\boldsymbol{i}}$ \\
\hline 1 & 2 & 0.1 & 4.4 & -15.6 & 12 & 1 \\
\hline 2 & 0.25 & 10 & 6.15 & 0.570 & 0.12 & 1 \\
\hline
\end{tabular}

Table 1: Plant and controller parameters

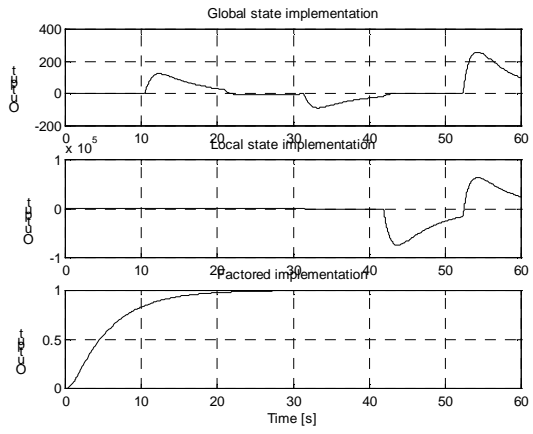

(a) Periodic switching between controllers every 10 seconds with a duty cycle of 0.5 .

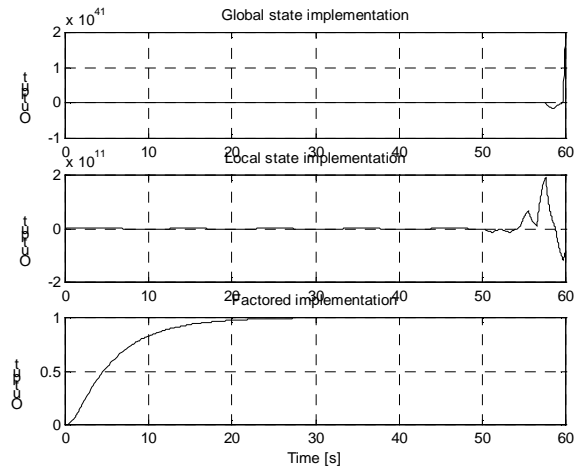

(b) Periodic switching beween controllers every 1 seconds with a duty cycle of 0.5 .

Figure 10: Response to unity input of various realisations

It is clear from the above plots that the qualitative response of each implementation is very different. For both switching sequences, the response of the local state and the global state controllers is unsatisfactory; in (b) the global state controller is unstable. This is a genuine switching instability; namely, both $A_{1}$ and $A_{2}$ are Hurwitz-stable, but as a result of switching instability occurs. The local state implementation is unstable in both (a) and (b). However, here we are switching between matrices that are themselves unstable (due to the integrator poles), and the instability that arises is as a result of conventional LTI instability mechanisms and switching. The response of the factored implementation is almost perfect. Not only is the system stable, but the switching is also completely unobservable 
from the output of the feedback loop; see work in (Shorten and O'Cairbre, 2002) for initial stability results for local state controllers.

\section{Remark: Global state, local state and factored implementations, and a new problem in switching}

The fact that controller realisation can have such an impact on the performance of the closed loop system has profound implications for control design. An important question is to account for and exploit these differences for the design of control systems. Clearly, whenever all controllers share the same structure then the controller may be implemented in the form of a parameter varying system (state sharing), or by switching between the individual controllers (local state), and sometimes in the form of a factored implementation. In the global state case the controller has a single state, where as in the latter cases each of the controllers have local states. An obvious difference between the two cases is that the global state approach results in a control output that is continuous at the switching instants, whereas the local state approach results in a controller output that is discontinuous at the switching instants. Other differences between the two approaches is that the global state approach requires fewer states to implement, and that the global state approach results in a controller structure that is itself parameter varying and hence can be potentially destabilised by means of switching. In the above example, the controller parameters are identical in both cases and are given in table 1 . However, the state space realisations are quite different. The local state implementation results in a closed loop system that has a higher order than that of the global state implementation or of the factored state implementation.

\begin{tabular}{|c|c|c|}
\hline Implementation & Number of states & $\boldsymbol{R a n k}\left(\boldsymbol{A}_{\boldsymbol{1}}-\boldsymbol{A}_{\boldsymbol{2}}\right)$ \\
\hline Global state & 3 & 2 \\
\hline Local state & 5 & 2 \\
\hline Factored & 4 & 1 \\
\hline
\end{tabular}

Table 2

A more crucial observation concerns the rank of the matrix $\left(\mathbf{A}_{\mathbf{1}}-\mathbf{A}_{\mathbf{2}}\right)$; instability mechanisms for global state control system are excited via rank-2 perturbations, whereas instability in the factored implementation is excited via a rank-1 perturbation. While it is true to say that few stability results are known that are valid for rank-2 perturbed systems, the rank-1 case is well understood. In fact, it is shown in (Shorten and O'Cairbre, 2002) that system matrices that share $n-1$ common eigenvalues, and which satisfy $\operatorname{rank}\left(\mathbf{A}_{\mathbf{1}}-\mathbf{A}_{\mathbf{2}}\right)=1$ result in asymptotically stable switching systems.

Example 4 illustrates another very important characteristic of the factored implementation; namely that the switching action is completely unobservable from the output of the control system. We note that it is impossible to realise such a design specification with the global state implementation (without state re-initialisation), or with the local state controllers (with unstable local controllers). The poor performance of the control structure is due to the fact that after switching the controller must find its equilibrium condition under the dynamics of the closed loop. Such effects can be avoided by employing local state controllers (with stable controllers). In this case the new equilibrium condition can be found by switching between the controller outputs. A simple condition for unobservability of the switching action, for a constant command input, is that the steady state gain between the command and the output is constant. While it is relatively easy to design local state controllers (where the controllers are stable) to achieve this property for constant input signals (Shorten 96), the question of whether or not this is possible for arbitrary input signals has not yet been explored. It is therefore of interest to pose the following question:

Observability of switching action: Given a class of input signals $u \in \vartheta$, for what class of switching systems.

$$
\begin{aligned}
\Omega: \quad \dot{x} & =A(t) x+B(t) u, \quad x\left(t_{0}\right)=x_{0}, \\
& \mathrm{y}=\mathrm{C}(\mathrm{t}) \mathrm{x},
\end{aligned}
$$

is the switching action unobservable, given access to the output y only? 


\section{A Benchmark System}

In many control applications, the controller includes switches and its performance depends directly on the successful resolution of the issues discussed above. Indeed, these aspects of the controller design may be the most important. One such application is the control of large-scale grid-connected variablespeed pitch-regulated wind turbines. The wind turbine system essentially consists of a rotor, a lowspeed shaft, a gearbox, a high-speed shaft and a generator. The rotor blades pitch about their longitudinal axis. A much-simplified representation of the dynamics of a $1 \mathrm{MW}$ three-bladed machine is proposed as a benchmark switched system. A block diagram representation of this system is depicted in Figure 11. The characteristics of the turbine component sub-systems and specification of the control design task are described below (see Leithead \& Connor 2000a and references therein for detailed, generic derivation and validation of a more complete description).

\subsection{Plant dynamics}

(i) Power train

The combined dynamics of the drive-train and generator are essentially linear and, together, are modelled by

$$
\dot{\mathbf{x}}_{\mathrm{p}}=\mathbf{A}_{\mathrm{p}} \mathbf{x}_{\mathrm{p}}+\mathbf{B}_{\mathrm{p}} \mathbf{T}
$$

with

$$
\begin{aligned}
\mathbf{x}_{\mathbf{p}} & =\left[\begin{array}{lll}
\Omega_{L D} & T_{L S} & \Omega_{L S}
\end{array}\right]^{T}, \mathbf{T}=\left[\begin{array}{ll}
T_{L D} & T_{t r}
\end{array}\right]^{T} \\
\mathbf{A}_{\mathbf{p}} & =\left[\begin{array}{ccc}
-\gamma_{2} / I_{2} & 1 / N I_{2} & 0 \\
-\hat{K}_{1} / N & 0 & \hat{K}_{1} \\
\left(\gamma / N-\gamma \hat{K}_{1} /\left(N^{3} K_{2}\right)\right) / I_{1} & -1 / I_{1} & -\left(\gamma_{1}+\gamma-\gamma \hat{K}_{1} /\left(N^{2} K_{2}\right)\right) / I_{1}
\end{array}\right], \mathbf{B}_{\mathbf{p}}=\left[\begin{array}{cc}
-1 / I_{2} & 0 \\
0 & 0 \\
0 & 1 / I_{1}
\end{array}\right]
\end{aligned}
$$

where $\mathrm{T}_{\mathrm{LS}}$ is the torque on the low speed shaft, $\Omega_{\mathrm{LS}}$ is the speed of the low-speed shaft, $\Omega_{\mathrm{HS}}$ is the speed of the high-speed shaft, $\Omega_{\mathrm{LD}}$ is the generator speed, $\mathrm{T}_{\mathrm{rtr}}$ is the torque generated by the rotor, $\mathrm{T}_{\mathrm{LD}}$ is the generator reaction torque (set via power electronics). The parameter values are $\mathrm{N}=58$; $I_{1}=1.0295 \times 10^{6} ; I_{2}=42.82 ; K_{1}=1.0106 \times 10^{8} ; K_{2}=4.85 \times 10^{6} ; \gamma_{1}=1.5176 \times 10^{4} ; \gamma_{2}=4.5112 ; \gamma=2.6538 \times 10^{5}$, $\hat{\mathrm{K}}_{1}=\mathrm{K}_{1} /\left(1+\frac{\mathrm{K}_{1}}{\mathrm{~K}_{2} \mathrm{~N}^{2}}\right)$. (It should be noted that the value of $\gamma_{1}$ embodies the linear component of the damping introduced by aerodynamic effects). The transfer function relationship, equivalent to (6), is

$$
\Omega_{L D}(s)=\frac{-\left(0.02335 s^{2}+0.00633 s+2.279\right) T_{L D}(s)+0.0393 T_{r t r}(s)}{s^{3}+0.3764 s^{2}+794.9 s+20.57}
$$

(ii) Pitch actuator

The pitch actuator position is physically constrained to be greater than or equal to zero degrees. The actuator dynamics may be neglected for analysis purposes. For completeness, however, it is noted that these are

$$
\begin{aligned}
\dot{\mathbf{x}}_{\mathrm{z}}=\mathbf{A}_{\mathrm{a}} \mathbf{x}_{\mathrm{a}}+\mathbf{B}_{\mathrm{a}} \hat{\mathrm{p}} & , \quad \mathrm{p}=\mathbf{C}_{\mathrm{a}} \mathbf{x}_{\mathrm{a}} \\
\mathbf{A}_{\mathrm{a}} & =\left[\begin{array}{ccc}
0 & 1 & 0 \\
-526.62 & -28.57 & -1022.73 \\
v(\hat{\mathrm{p}}) & 0 & 0
\end{array}\right], \quad \mathbf{B}_{\mathrm{a}}=\left[\begin{array}{c}
0 \\
1022.73 \\
0
\end{array}\right], \quad \mathbf{C}_{\mathrm{a}}=\left[\begin{array}{lll}
0 & 0 & 1
\end{array}\right]
\end{aligned}
$$

where $\mathrm{p}$ and $\hat{\mathrm{p}}$ are, respectively, the actual and demanded blade pitch angles and $v(\hat{\mathrm{p}})= \begin{cases}1 & \hat{\mathrm{p}}>0 \\ 0 & \hat{\mathrm{p}} \leq 0\end{cases}$ represents the position constraint. 


\section{(iii) Aerodynamics}

By suitably augmenting the plant (see Appendix), the aerodynamic torque, $\mathrm{T}_{\mathrm{rtr}}$, generated by the rotor may be approximately modelled by

$$
\mathrm{T}_{\mathrm{rtr}}=\mathrm{K}_{\mathrm{V}} \mathrm{V}^{2}-\mathrm{K}_{\mathrm{p}} \Delta\left(\mathrm{p}, \Omega_{\mathrm{LS}}\right) \mathrm{p}
$$

where $\mathrm{V}$ is the effective wind speed, see below, and $\Delta\left(\mathrm{p}, \Omega_{\mathrm{LS}}\right)$ is an unknown gain (representing uncertainty in the aerodynamic characteristics) with nominal value of unity, $K_{p}=29500.0$ and $\mathrm{K}_{\mathrm{V}}=3200.0$.

\section{(iv)Wind disturbance}

The rotor interacts with a complex spatially and temporally varying wind-field. However, the windfield may be represented by a single wind speed constant over the rotor disk, the effective wind speed. It should be noted that the spectral characteristic of this effective wind speed is very different from that of a point wind speed. For a given mean wind speeds $V_{\text {mean }}$, a suitable model for the effective wind speed is the linear stochastic equation

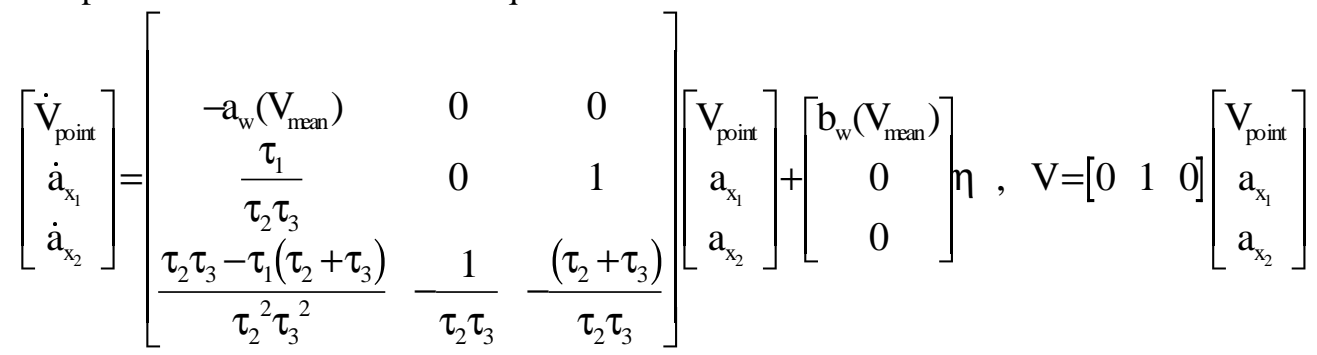

where $\tau_{1}=\beta / \sqrt{2}, \tau_{2}=\tau_{1} \sqrt{\mathrm{a}}, \tau_{3}=\beta / \sqrt{\mathrm{a}}, \beta=33.8 / \mathrm{V}_{\text {mean }}, \mathrm{a}=0.55$ and $\eta$ is Gaussian white noise with zero mean and unity variance. Suitable values for $a_{w}$ and $b_{w}$ (capturing the dependence on the mean wind speed of the spectral characteristics of the effective wind speed) are given in Table 3 ,

\begin{tabular}{|l|l|l|}
\hline $\mathrm{V}_{\text {mean }}(\mathrm{m} / \mathrm{s})$ & $\mathrm{a}_{\mathrm{w}}$ & $\mathrm{b}_{\mathrm{w}}$ \\
\hline 12 & 0.068 & 0.88 \\
16 & 0.092 & 1.37 \\
20 & 0.114 & 1.77 \\
\hline
\end{tabular}

Table 3 Mean wind speed dependent parameters for wind disturbance model.

\subsection{Design task: control requirements}

The overall objective of the controller is to maximise energy production, whilst working within actuator operational limits and minimising the extreme loads and associated fatigue damage on the turbine structure and drive train. This is a disturbance rejection task.

\section{(i) Measured variables}

Measurements are available of (i) the instantaneous power P (i.e. the product $\mathrm{T}_{\mathrm{LD}} \Omega_{\mathrm{LD}}$ ) and (ii) the generator speed $\Omega_{\mathrm{LD}}$. The sensor dynamics can be assumed negligible, as is measurement noise. Note that the effective wind speed, $\mathrm{V}$, cannot be measured.

\section{(ii) Manipulated variables}

The controller is able to adjust (i) the blade pitch angle and (ii) the generator reaction torque, $T_{L D}$.

\section{(iii) Physical constraints}

Controller activity is constrained by two main operational factors. Firstly, the controller is required to operate within the constraints of the available actuator. In addition to the constraint that the blade angle must be non-negative, the machine considered here employs a hydraulic pitch actuator for which the relevant measure of actuator activity is the rate of change of the blade pitch angle. The standard 
deviation of the rate of change of the pitch angle reflects the actuator activity over the medium and long term and is required to remain less than $(0.4 \mathrm{~V}-1.8) \mathrm{deg} / \mathrm{s}$ over the operating range of mean wind speeds up to $24 \mathrm{~m} / \mathrm{s}$ (the dependence of the bound on wind speed is associated with the augmentation of the plant to compensate for the aerodynamic nonlinearity - see Appendix). Secondly, in order to avoid exciting structural resonances and to remain within design loadings, the turbine is not to be continuously operated (i.e. in steady state) at rotor speeds above $2.72 \mathrm{rads} / \mathrm{sec}$. Of course, fluctuations in the mean wind speed induce transients (about the steady state operating point) in the rotor speed. These transients must remain strictly less than $3.264 \mathrm{rads} / \mathrm{sec}$ under the normal range of operating conditions. The former limit is denoted $\Omega_{\mathrm{LS}}^{\max c o n t}$ and the latter $\Omega_{\mathrm{LS}}^{\max }$. The generator reaction torque, $\mathrm{T}_{\mathrm{LD}}$, must be positive (to avoid "motoring") and the generator is not to be operated continuously (i.e. in steady state) above a level, $\mathrm{P}_{\text {rated }}$, of $1 \mathrm{MW}$.

\section{(iv) Robustness}

The uncertainty in the plant dynamics is primarily associated with the rotor aerodynamics. In addition to the use of a relatively crude aerodynamic model for control design purposes, the rotor aerodynamics typically exhibit considerable variation during normal operation (associated with, in particular, the accumulation of environmental deposits on the blade surfaces). The closed-loop system is therefore required to remain stable for arbitrary time variations in the uncertain gain $\Delta$ in the interval $[0.5,2]$.

\section{(v) Operational Requirement}

The overall objective of the controller is to maximise energy production, whilst working within the operational limits of the turbine, and minimising the peak loadings experienced. While the wind is highly stochastic, initial insight into this requirement can be gained by considering the situation when the wind is steady and the turbine is in equilibrium. Three operating modes can be identified.

1. Energy capture limited by available wind energy

2. Energy capture limited by rotor speed constraints

3. Energy capture limited by generator rating

\section{(vi) Performance assessment}

Performance is measured as follows (the approach adopted is semi-empirical in view of the complex, stochastic nature of the wind disturbance; see Leithead \& Connor 2000b). Time histories of the controlled system are collected for turbulent wind conditions with mean wind speeds $\mathrm{V}_{\text {mean }} \in\{5,8,10,12,14,16,18,20,22\} \mathrm{m} / \mathrm{s}$. The time histories are each of 600 seconds duration (after discarding the initial 20 seconds to allow the system to settle down) and are partitioned into 10 second intervals. The short-term mean wind speed, mean power, mean generator torque, peak power, maximum rotor speed, minimum generator torque and standard deviation of pitch actuator velocity are determined for each interval. This interval data is sorted into $1 \mathrm{~m} / \mathrm{s}$ wide bins according to the shortterm mean wind speed. Let $\mathrm{V}_{\mathrm{i}}$ denote the centre wind speed of the $\mathrm{i}^{\text {th }}$ bin. The average of the mean power data in the $i^{\text {th }}$ bin is a measure of energy capture at wind speed $V_{i}$. Let $\mu_{i}$ denote the average of the peak power data in the $\mathrm{i}^{\text {th }}$ bin. Then $\mu_{\mathrm{i}}$ is a measure of the peak load experienced by the wind turbine at wind speed $\mathrm{V}_{\mathrm{i}}$. Energy capture is to be maximised and peak loads minimised, subject to operating constraints. Note that a high penalty is placed on peak loads during above rated operation as these are related to fatique and therefore the impact is dominated by the peak values. A penalty is placed on sub-optimal energy capture during below rated operation as in practice this would lead to operation in the aerodynamic stall regime (not modelled here). Similar calculations for the maximum rotor speed data, minimum generator torque data and pitch actuator velocity data provides, respectively, an upper bound on $\Omega_{\mathrm{LS}}$ (required to be less than $\Omega_{\mathrm{LS}}^{\max }$ ), a lower bound on $\mathrm{T}_{\mathrm{LD}}$ (required to be positive) and an upper bound on pitch actuator velocity. A further, deterministic, extreme gust is employed to confirm the ability of the controller to maintain operation within the allowed rotor speed limits. This gust is a pulse with an initial wind speed of $22 \mathrm{~m} / \mathrm{s}$, falling to $12 \mathrm{~m} / \mathrm{s}$ over 10 seconds and then returning to $22 \mathrm{~m} / \mathrm{s}$.

\subsection{Discussion}

The wind turbine system described above is clearly a switched system. Because of the lower limit on rotor pitch angle, control via pitch is intermittent. This intermittent action can be interpreted as a 
switching action. Additionally, the changes between the three modes of control operation require switches. These switches are activated very frequently and randomly by the stochastic variation in wind speed. Nevertheless, the robust stability of the controlled system must be maintained. Furthermore, the transients associated with the switches can very easily cause the performance requirements to be breeched. Hence, suppression, indeed elimination, of the switching transients is critical. In contrast to the examples in Section 2, which are not untypical of those considered elsewhere in the literature, the wind turbine system, having multiple switches and higher order dynamics, is rather more complex as indeed are many switched systems in practice.

\section{Baseline Design: Classical Controller}

Successful solutions to the pitch-regulated variable speed wind turbine control design task are known and are described in Connor and Leithead (1996). A similar controller for the wind turbine system presented above is described in this section.

\subsection{Baseline controller}

Clearly, the performance requirements cannot be met with a single linear controller. Reflecting the natural division of the turbine operation into separate modes, the approach standard in commercial practice is adapted whereby in each mode of operation a particular control action is chosen and an individual controller is designed. Subsequently, the individual controllers are integrated to obtain a full envelope controller.

\section{(i) Operating mode 1}

The controller is configured to regulate rotor speed to maximise energy capture by adjusting the generator reaction torque whilst maintaining the blade pitch angle at zero. Under nominal steady state conditions, the maximum power is generated when $T_{L D}=K_{V} V^{2} / 2 N$. Unfortunately, as the effective wind speed $\mathrm{V}$ is not measurable, direct regulation of $\mathrm{T}_{\mathrm{LD}}$ to meet this equality is impossible. Instead, an indirect approach must be used. In steady conditions generating maximum power, the generator speed is $\Omega_{\mathrm{LD}}=\mathrm{NK}_{\mathrm{V}} \mathrm{V}^{2} / 2\left(\gamma_{1}+\mathrm{N}^{2} \gamma_{2}\right)$. That is, $\mathrm{K}_{\mathrm{v}} \mathrm{V}^{2}=2\left(\gamma_{1}+\mathrm{N}^{2} \gamma_{2}\right) \Omega_{\mathrm{LD}} / \mathrm{N}$. Consider, therefore, the control law

$$
T_{L D}=C_{L D} \frac{\left(\gamma_{1}+N^{2} \gamma_{2}\right)}{N^{2}} \Omega_{L D}
$$

where

$$
\mathrm{C}_{\mathrm{LD}}=\frac{1}{0.05 s+1}
$$

provides additional roll-off to suppress the resonance in the turbine drive-train. (Note that in actual wind turbine controllers it is more usual to select $\mathrm{T}_{\mathrm{LD}}$ proportional to $\Omega_{\mathrm{LD}}{ }^{2}$ rather than $\Omega_{\mathrm{LD}}$. The linear law used here approximates the usual quadratic law, in accordance with the simplified aerodynamic model used).

\section{(ii) Operating mode 2}

The controller is configured to regulate rotor speed, $\Omega_{\mathrm{LS}}^{\max c o n t}$, at a constant value by adjusting the generator reaction torque while maintaining the blade pitch angle at zero. Using classical loopshaping techniques, the controller transfer function is designed to incorporate integral action to ensure rejection of changes in mean wind speed. The controller designed is

$$
T_{L D}=C_{1}\left(N \Omega_{L S}^{\max c o n t}-\Omega_{L D}\right)+C_{L D} \frac{\left(\gamma_{1}+N^{2} \gamma_{2}\right)}{N^{2}} \Omega_{L D}
$$

where $\mathrm{C}_{1}$ and $\mathrm{C}_{\mathrm{LD}}$ denote linear dynamics with transfer functions 


$$
C_{1}=\frac{1200(5 s+1)}{s\left(s^{2}+4 s+14\right)(0.05 s+1)}
$$

Observe that, with the aim of simplifying the overall design, the mode 2 controller (15) is obtained by suitably augmenting the existing mode 1 controller, (13). The gain margin is $9.67 \mathrm{~dB}$, the phase margin is $56.51^{\circ}$ and the cross-over frequency is $1.30 \mathrm{rad} / \mathrm{s}$.

\section{(iii) Operating mode 3}

The controller is configured to regulate rotor speed at a constant value, $\Omega_{\mathrm{LS}}^{\max c o n t}$, by adjusting the blade pitch angle whilst keeping a fixed generator reaction torque, $T_{\text {ref }}$. Neglecting, for the moment, the high frequency drive-train resonance, the plant dynamics (6) may be simplified to

$$
\dot{\Omega}_{\mathrm{DD}}=\frac{\mathrm{NT}_{\mathrm{tt}}-\mathrm{N}^{2} \mathrm{~T}_{\mathrm{D}}-\gamma_{1} \Omega_{\mathrm{D}}}{\mathrm{I}_{1}}=\frac{-\mathrm{NK}_{\mathrm{p}} \mathrm{p}-\mathrm{N}^{2} \mathrm{~T}_{\mathrm{DD}}-\gamma_{1} \Omega_{\mathrm{D}}}{\mathrm{I}_{1}}+\frac{\mathrm{NK}_{\mathrm{V}}}{\mathrm{I}_{1}} \mathrm{~V}^{2}
$$

Evidently, the aerodynamic torque and generator torque are matched in the sense that they enter the equation in the same manner, albeit with a gain difference of $\mathrm{N}$. Hence, despite the physical structure of the system being quite different in modes 2 and 3 (in mode 2 control action applied via the generator torque alone, while in mode 3 the system is configured as MIMO with control action applied via both the pitch angle and generator torque), in terms of the plant dynamic characteristics these modes are closely related.

Using classical loop-shaping techniques, the controller is designed as

$$
\hat{\mathrm{p}}=\frac{\mathrm{N}}{\mathrm{K}_{\mathrm{p}}} \mathrm{C}_{2}\left(\mathrm{~N} \Omega_{\mathrm{LS}}^{\max c o n t}-\Omega_{\mathrm{LD}}\right)+\frac{\left(\gamma_{1}+\mathrm{N}^{2} \gamma_{2}\right)}{\mathrm{N}^{2}} \Omega_{\mathrm{LD}}
$$

where $\mathrm{C}_{2}$ denotes linear dynamics with transfer function

$$
C_{2}=\frac{1200(5 s+1)}{s\left(s^{2}+4 s+14\right)}
$$

Observe that, at least at low frequencies, the controller transfer function is closely related to that employed in mode 2, reflecting the similarity in plant dynamics in modes 2 and 3 noted previously. The gain margin is $9.84 \mathrm{~dB}$, the phase margin is $59.63^{\circ}$ and the cross-over frequency is $1.33 \mathrm{rad} / \mathrm{s}$.

\section{(iv) Full envelope controller}

It remains to integrate the separate mode 1, 2 and 3 controllers to produce a full-envelope controller implementation. Firstly, it is noted that the mode 2 and 3 controllers are designed to directly augment the mode 1 controller, thereby simplifying implementation. Secondly, the mode 2 and 3 controllers possess similar low frequency dynamics. The latter can be made explicit by partitioning the mode 2 and 3 controller transfer functions as

$$
\mathrm{C}_{1}=\mathrm{C}_{\mathrm{LD}} \mathrm{C}_{\text {low }} \quad, \quad \mathrm{C}_{2}=\mathrm{C}_{\text {low }}
$$

where

$$
C_{\text {low }}=\frac{1200(5 s+1)}{s\left(s^{2}+4 s+14\right)}, \quad \mathrm{C}_{\mathrm{LD}}=\frac{1}{(0.05 s+1)}
$$

During mode 1 operation, the integrator in $\mathrm{C}_{\text {low }}$ can "wind up" resulting in excessive transients following a transition from mode 1 to mode 2 operation. Transients may also be associated with the other low frequency dynamics elements of $\mathrm{C}_{\text {low }}$. Following Leith \& Leithead (1997), and similarly to a number of popular anti-wind up approaches, this issue is addressed here by partitioning $\mathrm{C}_{\text {low }}$ as $\mathrm{C}_{\text {low }}=\mathrm{C}_{\mathrm{o}} \mathrm{C}_{\mathrm{i}}$ where 


$$
C_{i}=-15.625 \frac{(5 s+1)}{s\left(s^{2}+4 s+14\right)}, C_{o}=76.8
$$

and enclosing the dynamics $C_{i}$ within a minor feedback loop during mode 1 operation. The partitioning into $\mathrm{C}_{\mathrm{o}}$ and $\mathrm{C}_{\mathrm{i}}$ is selected such that the bandwidth of the minor loop is similar to that of the closed-loop system during mode $2 / 3$ operation. Switching from mode 2 to mode 3 operation is based on the generator reaction torque. The resulting full envelope controller implementation is shown in Figure 12. Observe that the switches within the controller are formulated as continuous (but nondifferentiable) nonlinear functions of the switch input. Hence, the admissible switching sequences are immediately evident from the block diagram. The constant $\mathrm{T}_{\text {ref }}$ is defined by

$$
\mathrm{T}_{\text {ref }}=\mathrm{P}_{\text {rated }} / \mathrm{N} \Omega_{L S}^{\max c o n t}
$$

that is, $T_{\text {ref }}$ is the generator torque at which rated power is developed when operating at rated speed. This is used within the controller in a straightforward manner as a threshold on internal signals to determine the required mode of operation.

Remark: The following state-space realisations are employed.

$$
\begin{gathered}
\mathrm{C}_{\mathrm{LD}}: \quad \dot{\mathbf{x}}_{\mathrm{LD}}=\mathbf{A}_{\mathrm{LD}} \mathbf{x}_{\mathrm{LD}}+\mathbf{B}_{\mathrm{LD}} \mathrm{T}_{1}, \mathrm{~T}_{\mathrm{LD}}=\mathbf{C}_{\mathrm{LD}} \mathbf{x}_{\mathrm{LD}} \\
\mathrm{C}_{\mathrm{i}} \mathrm{C}_{\mathrm{o}}: \quad \dot{\mathbf{x}}_{\text {low }}=\mathbf{A}_{\text {low }} \mathbf{x}_{\text {low }}+\mathbf{B}_{\text {low }}\left(\left(\mathrm{N} \Omega_{\mathrm{LS}}^{\text {mxxoort }}-\Omega_{\mathrm{LD}}\right)+\mathrm{B}_{\mathrm{o}} \mathrm{T}_{1}\right), \mathrm{T}_{2}=\mathbf{C}_{\text {low }} \mathbf{x}_{\text {low }}
\end{gathered}
$$

where

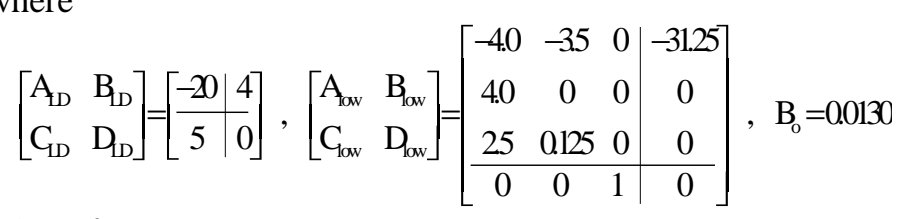

\section{(v) Performance}

Many different ways of integrating the individual controllers for the separate modes is possible but it is emphasised that the performance is sensitive to the way in which this integration is achieved. The particular full-envelope controller described above achieves good performance and fully meets the performance specification. Although its performance can be improved upon, the required modifications increase its complexity. Hence, to facilitate analysis, this baseline controller represents a suitable compromise between performance and complexity. Detailed performance plots are shown in Figure 13. A Simulink model of the plant and controller is available at www.hamilton.may.ie,.

\subsection{Analysis challenges}

The baseline controller design immediately creates a number of analysis tasks. While analysis of the robust stability and performance of the full-envelope closed-loop system is required, the local pairwise analysis of the operating modes nominal stability presents a task of sufficient difficulty for present purposes. While reading this section, it is important to keep in mind that we are dealing with mode 1 /mode 2 switching and mode $2 /$ mode 3 switching separately. We do not consider switching between mode 1 and mode 3 .

\section{(i) Stability of mode 1/2 operation}

During operation encompassing modes 1 and 2, the closed-loop dynamics (neglecting the additive wind disturbance and the constant reference inputs to the controller) are

$$
\dot{\mathbf{x}}=\mathbf{A}_{\mathrm{i}} \mathbf{x} \quad, \quad \mathrm{i} \in\{1,2\}
$$




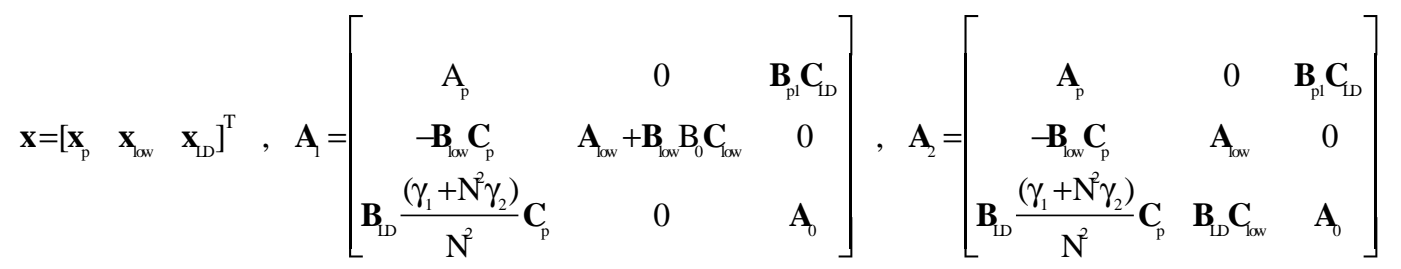

where $\mathbf{B}_{\mathbf{p} 1}$ denotes the first column of $\mathbf{B}_{\mathbf{p}}, \mathbf{C}_{\mathbf{p}}=\left[\begin{array}{lll}1 & 0 & 0\end{array}\right]$.

One standard approach to stability analysis of a switched system of the form (26) is to search for a common quadratic Lyapunov function (CQLF), $\mathbf{x}^{\mathrm{T}} \mathbf{P x}$. That is, for a matrix $\mathbf{P}$ such that

$$
\mathbf{P}>0, \quad \mathbf{A}_{1}^{T} \mathbf{P}+\mathbf{P} \mathbf{A}_{1}<0, \quad \mathbf{A}_{2}^{T} \mathbf{P}+\mathbf{P} \mathbf{A}_{2}<0
$$

The existence of such a matrix guarantees the exponential stability of (26) for all switching sequences (e.g. see Liberzon \& Morse 1999, Shorten \& Narendra 1998b and references therein). As mentioned in section 3, the existence of a CQLF can be established numerically using software for solving LMIs. A direct search for a matrix $\mathbf{P}$ satisfying the inequalities (28) performed using the LMI toolbox in MATLAB fails to establish the existence of a CQLF for the system (26). In fact, the toolbox finds the system of LMIs (28) to be marginally feasible, meaning that it is unable to find a CQLF while also being unable to definitively rule out the existence of one. However it can be confirmed using the following direct analytic arguments that no CQLF exists for this system. A necessary condition for the existence of a CQLF is that the matrix pencils

$$
\begin{aligned}
& \alpha \mathbf{A}_{1}+(1-\alpha) \mathbf{A}_{2} \\
& \alpha \mathbf{A}_{1}+(1-\alpha) \mathbf{A}_{2}^{-1}
\end{aligned}
$$

must be Hurwitz for $\alpha \in[0,1]$. (Shorten and Narendra, 1999). This clearly implies that both pencils must be non-singular for $\alpha \in[0,1]$. However, it follows from the fact that the matrix product $\mathbf{A}_{1} \mathbf{A}_{2}$ has negative real eigenvalues that the pencil $\alpha \mathbf{A}_{1}+(1-\alpha) \mathbf{A}_{2}^{-1}$ is not Hurwitz for some $\alpha \in[0,1]$. Thus, the system (26) cannot have a CQLF. For more details on this analysis consult (Mason, Shorten \& Leith, 2001) and the references therein. This is an example of the situation described in section 3, where a numerical approach to stability analysis can fail to provide the correct answer where alternative direct arguments succeed.

While the forgoing analysis considers quadratic Lyapunov functions, LMI based analysis may be extended to encompass searching for the existence of a class of piecewise-quadratic Lyapunov functions (Johansson \& Rantzer 1998). The state-space is divided into two regions or cells, with mode 1 effective in cell $1\left(\mathrm{x}_{6}<0\right)$ and mode 2 effective in cell $2\left(\mathrm{x}_{6} \geq 0\right)$. To establish stability via piecewise methods, a Lyapunov function of the form

$$
V(x)= \begin{cases}\mathbf{x}^{\mathbf{T}} \mathbf{P}_{1} \mathbf{x} & \text { in cell } 1 \\ \mathbf{x}^{\mathbf{T}} \mathbf{P}_{2} \mathbf{x} & \text { in cell } 2\end{cases}
$$

is sought, where the matrices $\mathbf{P}_{\mathrm{i}}$ are parameterised so as to ensure that the function is continuous across the boundaries between the cells. Namely, following Johansson \& Rantzer (1998) the matrices $\mathbf{P}_{\mathrm{i}}$ are parameterised as $\mathbf{P}_{\mathrm{i}}=\mathbf{F}_{\mathrm{i}}^{\mathrm{T}} \mathbf{T F}_{\mathrm{i}}$, i=1,2 where the matrix $\mathbf{T}$ is to be determined and $\mathbf{F}_{1} \mathbf{x}=\mathbf{F}_{2} \mathbf{x}$ on the shared cell boundary. Note that these matrices are not uniquely determined by the partition. This formulation relaxes the requirement of a common quadratic Lyapunov function in two ways. Firstly, we do not require a single positive definite matrix $\mathbf{P}$ to simultaneously satisfy $\mathbf{A}_{\mathrm{i}}{ }^{\mathrm{T}} \mathbf{P}+\mathbf{P} \mathbf{A}_{\mathrm{i}}<\mathbf{0}$ for each $\mathrm{i}$. Secondly, when implementing the search for such a function as a system of LMIs, $\mathbf{x}^{\mathrm{T}}\left(\mathbf{A}_{\mathrm{i}}{ }^{\mathrm{T}} \mathbf{P}_{\mathbf{i}}+\mathbf{P}_{\mathbf{i}} \mathbf{A}_{\mathbf{i}}\right) \mathbf{x}$ is not required to be negative for all non-zero $\mathbf{x}$ but only for those $\mathbf{x}$ in the cell $\mathrm{i}$ where the dynamics are given by the system matrix $\mathbf{A}_{\mathrm{i}}$. As discussed in section 3, the problem of finding a PQLF for the 
system can be formulated as a feasibility problem for a system of LMIs and solved numerically (Johansson \& Rantzer 1998). The PWLTOOL package (Hedlund \& Johansson, 1999) for analysis of piecewise linear systems was used to test for the existence of a PQLF. Unfortunately, PQLF analysis also failed to establish the stability of the system. Apropos the use of piecewise quadratic methods, it is important to note that we select a partition of the state space, and search for a Lyapunov function that is piecewise quadratic with respect to that partition. This choice of partition is not unique. In particular, we are not constrained to use the partition suggested by the dynamics of the system. In fact, it is possible that no PQLF exists for one choice of partition, while an alternative partition may yield a PQLF for the same system. Thus it is difficult to give a definitive negative answer to the PQLF existence question - the fact that there is no PQLF for one choice of partition does not imply that the stability of the system cannot be established via a piecewise quadratic Lyapunov function. Less conservative results can be obtained using harmonic balance techniques at the price of reduced rigour. Harmonic balance analysis, along the lines presented in Example 2, indicates stability of the switched system. This result is supported by extensive nonlinear simulations. In view of the foregoing results, the challenge is evidently to derive stability analysis methods capable of providing tight, yet soundlybased, results for the type of system considered here. The lack of non-conservative stability analysis methods is surely one of the major outstanding issues in the control field today. Of particular interest in the present example is the close link between switching system methods and anti-windup techniques which it exemplifies. The existence of a relationship between anti-windup and bumpless transfer methods is often noted at an intuitive level but it is less often discussed in the context of the rigorous stability analysis of switched systems. The key role of the conditioning loop within the controller here in avoiding prolonged transient excursions following switching is readily verified by simulation testing (see also the examples studied in Leith \& Leithead (1997) in the context of constant-speed wind turbines) yet despite much work in the switched system and anti-windup fields current techniques for the design of such loops remain largely ad hoc in nature.

\section{(ii) Stability of mode 2/3 operation}

Assume, for the moment, that the filter $\mathrm{C}_{\mathrm{LD}}$ has sufficiently fast dynamics that the switch within the controller can be moved to the input of $\mathrm{C}_{\mathrm{LD}}$. Under these conditions, the switches in the pitch angle and generator torque channels of the controller operate synchronously. It follows that the closed-loop dynamics (neglecting the additive wind disturbance and the constant reference inputs to the controller) are then

$$
\dot{\mathbf{x}}=\mathbf{A}_{i} \mathbf{x}, \quad i \in\{2,3\}
$$

with

$$
\mathbf{A}_{3}=\left[\begin{array}{ccc}
\mathbf{A}_{\mathbf{p}}-\mathbf{B}_{\mathbf{p}_{2}} \frac{\left(\gamma_{1}+N^{2} \gamma_{2}\right)}{N} \mathbf{C}_{p} & -\mathbf{B}_{\mathbf{p}_{2}} N \mathbf{C}_{l o w} & 0 \\
-\mathbf{B}_{l o w} \mathbf{C}_{\mathbf{p}} & \mathbf{A}_{l o w} & 0 \\
\mathbf{B}_{L D} \frac{\left(\gamma_{1}+N^{2} \gamma_{2}\right)}{N^{2}} \mathbf{C}_{\mathbf{p}} & \mathbf{B}_{L D} \mathbf{C}_{l o w} & \mathbf{A}_{L D}
\end{array}\right]
$$

where $\mathbf{B}_{\mathbf{p} 2}$ denotes the second column of $\mathbf{B}_{\mathrm{p}}$. As in section 5.1, the stability of this system may be analysed using CQLF techniques. A direct search performed using the LMI toolbox in MATLAB, successfully establishes the existence of a CQLF and thereby the exponential stability of the system. The validity of the foregoing synchronicity assumption is, however, very debatable. Consider therefore relaxing this assumption that the torque switch may be moved to the input of the $\mathrm{C}_{\mathrm{LD}}$ dynamics. The input to the torque channel switch is simply the input to the pitch switch filtered by $\mathrm{C}_{\mathrm{LD}}$. The $\mathrm{C}_{\mathrm{LD}}$ dynamics are fast compared to the bandwidth of the closed-loop system and thus, while the switches in the pitch and torque channels are asynchronous, the asynchronicity can only exist transiently. Nevertheless, this change has a profound impact on the stability analysis. For CQLF-type stability analysis, the closed-loop system is now conventionally modelled as switching between four possible system matrices rather than two. The extra two matrices correspond to transient situations whereby the pitch and torque switches are either simultaneously active or simultaneously inactive. Equivalently, the closed-loop can be modelled as

$$
\dot{\mathbf{x}}=\mathbf{A x}+\mathbf{B K}(\mathbf{y}) \mathbf{y}, \mathbf{y}=\mathbf{C x}+\mathbf{D r}
$$

where 


$$
\begin{aligned}
& \mathbf{A}=\left[\begin{array}{ccc}
\mathbf{A}_{\mathbf{p}} & 0 & 0 \\
-\mathbf{B}_{\text {low }} \mathbf{C}_{\mathbf{p}} & \mathbf{A}_{\text {low }} & 0 \\
\mathbf{B}_{\mathrm{LD}} \frac{\left(\gamma_{1}+\mathrm{N}^{2} \gamma_{2}\right)}{\mathrm{N}^{2}} \mathbf{C}_{\mathrm{p}} & \mathbf{B}_{\mathrm{LD}} \mathbf{C}_{\mathrm{low}} & \mathbf{A}_{\mathrm{LD}}
\end{array}\right], \mathbf{B}=\left[\begin{array}{ll}
\mathbf{B}_{\mathbf{p}_{1}} & -\mathbf{B}_{\mathbf{p}_{2}} \mathrm{~K}_{\mathrm{p}}
\end{array}\right], \mathbf{C}=\left[\begin{array}{ccc}
0 & 0 & \mathbf{C}_{\mathrm{LD}} \\
\frac{\left(\gamma_{1}+\mathrm{N}^{2} \gamma_{2}\right)}{\mathrm{NK}_{\mathrm{p}}} \mathbf{C}_{\mathbf{p}} & \frac{\mathrm{NC}_{\mathrm{low}}}{\mathrm{K}_{\mathrm{p}}} & 0
\end{array}\right] \\
& \mathbf{D}=0, \mathbf{K}(\mathbf{y}) \in\left\{\left[\begin{array}{ll}
1 & 0 \\
0 & 0
\end{array}\right],\left[\begin{array}{ll}
0 & 0 \\
0 & 1
\end{array}\right],\left[\begin{array}{ll}
0 & 0 \\
0 & 0
\end{array}\right],\left[\begin{array}{ll}
1 & 0 \\
0 & 1
\end{array}\right]\right\}
\end{aligned}
$$

The stability of (32) can be assessed using direct CQLF search techniques.. However, owing to the integral action within the controller, the closed-loop system is not exponentially stable for the case where both switches are simultaneously inactive and the system is effectively operating open-loop. While this situation exists only as a transient condition in the actual system, this information is not embodied in the representation (32) used for stability analysis purposes. The lack of information in (32) regarding this aspect of the switching behaviour means that there exist switching sequences in (32), which are not feasible in the actual system. In particular, there exist switching sequences for which (32) is not exponentially stable. Hence, no method based on this representation can establish the stability of the actual system. The challenge, therefore, is to develop analysis techniques which, by taking greater account of the admissible switching sequences, are capable of establishing the stability of the mode $2 / 3$ regime of operation without the need for unrealistic assumptions such as that of synchronicity used above. It is noted that the manner in which the system in Figure 12 is formulated, whereby the switches are each SISO nonlinear functions of their input, is strongly structured yet rather general, thus providing an interesting class of systems for which the potential exists for developing analysis methods which are of wide application yet not unduly conservative.

\section{Concluding remarks}

In this paper we have discussed some of the issues that arise when designing switched linear control systems. We have also presented a benchmark regulation problem, and a solution to this problem in the form of a switched linear controller. The limitations of currently available analysis techniques are highlighted by means of this example.

\section{Acknowldegements}

This work was partially supported by the European Union funded research training network MultiAgent Control, HPRN-CT-1999-00107 ${ }^{1}$ and by the Enterprise Ireland grant SC/2000/084/Y.

\section{References}

Blondel, V, (2002), Switched systems that are periodically stable may be unstable, submitted for publication;

Boyd, S., El Ghaoui, L., Feron, E. and Balakrishnan, V., 'Linear Matrix Inequalities in System and Control Theory', Philadelphia, SIAM, 1994

Branicky, M. S. (1994), Stability of switched and hybrid systems, Proc. IEEE Conference on Decision and Control, Florida, pp. 3498-3503;

Branicky,M.S., (1998), Multiple Lyapunov functions and other analysis tools for switched and hybrid systems, IEEE Trans. Aut. Control, 43, pp. 475-482;

Connor, B., Leithead, W.E., (1996), Investigation of control strategy of VS45, DTI Report ETSU W/42/00310/REP;

\footnotetext{
${ }^{1}$ This work is the sole responsibility of the authors and does not reflect the European Union's opinion. The EU is not responsible for any use of data appearing in this publication.
} 
Curran, P., (1998), An overview of absolute stability theory, Proceedings of the 6th International Specialist Workshop on Nonlinear Dynamics of Electronic Systems, NDES'98, Budapest, Hungary, 16-18 July, 1998, pp. 63-72;

DeCarlo, R., Branicky, M., Pettersson, S and Lennartson, B., (2000), Perspectives and results on the stability and stabilizability of hybrid systems, IEEE Proceedings, Vol. 88(7), pp. 1069-1082;

Goodwin G. C., Graebe, S. F. and Salgado, M. E., (2001), Control System Design, Prentice Hall;

Khalil, (1992), Nonlinear Systems Analysis, Macmillan;

Johansson, M., Rantzer, A., (1998), Computation of piecewise quadratic Lyapunov functions for hybrid systems, IEEE Trans Aut. Cont., 43, pp. 555-559;

Leith, D.J., Leithead, W.E. ,(1996), Performance enhancement of wind turbine power regulation by switched linear control, Int. J. Control, 65, 555-572; (1997), Implementation of wind turbine controllers;

Leith, D.J., Leithead, W.E. (1999), Global gain-scheduling control for variable speed wind turbine, Int.J.Control, 66,349-380;

Leithead,W.E., Connor,B. (2000a) Control of Variable Speed Wind Turbines: Dynamic Models. Int. J. Contr., 73, 1173-1188;

Leithead,W.E., Connor,B. (2000b) Control of Variable Speed Wind Turbines: Design Task. Int. J. Contr., 73, 1189-1212;

Liberzon, D. and Morse, A. S., Basic problems in stability and design of switched systems, IEEE Control Systems Magazine, 19(5), 59-70, 1999;

Mori, Y., Mori, T.and Kuroe, Y., (1998), On a wide class of linear constant systems that have a common quadratic Lyapunov function, Proceedings of the $36^{\text {th }}$ IEEE Conference on Decision and Control 1998, pp. 2808-2809;

Mason, O., Shorten, R. and Leith, D., 'Evaluation of piecewise quadratic methods applied to the windturbine benchmark example', Technical Report number 01/11, Signals and Systems group, NUI Maynooth, 2001

Narendra, K. S. and Cheng Xiang, (2000), Adaptive control of discrete-time systems using multiple models, IEEE Transactions on Automatic Control, Vol. 45(9), pp. 1669-1686;

Narendra, K. S., Shorten, R. and Boskovich, J. (1996), Intelligent control using multiple models, Proceedings of the $9^{\text {th }}$ Yale Workshop on Adaptive and Learning Systems, pp. 186-196, New Haven;

Narendra, K. S. and Balakrishnan, J., (1994a), A common Lyapunov function for stable LTI systems with commuting A-matrices, IEEE Transactions on Automatic Control, Vol. 39(12), pp. 1669-1686;

Narendra, K. S. and Balakrishnan, J., (1994b) Improving transient response of adaptive control systems using multiple models and switching, IEEE Trans. Aut. Control, 39, pp.1861-1866;

Pettersson, S. and Lennartson, B., 'LMI for stability and robustness of hybrid systems', in Proceedings of the American Control Conference, June 1997

Pyatnitski, E. S. and Rapoport, L. B., (1992), Periodic motion and tests for absolute stability on nonlinear nonstationar systems, Automation and Remote Control, Vol. 52(10), pp. 1379-1387; 
Shorten, R., (1996), A study of hybrid dynamical systems with application to automotive control, Ph.D. thesis, National University of Ireland;

Shorten,R.N., Narendra,K.S., (1999), Necessary and sufficient conditions for the existence of a common quadratic Lyapunov function for two stable second order linear time-invariant systems, Proc. 1999 Am. Control Conf., pp. 1410-1414;

Shorten,R.N., Narendra,K.S., (1998a), On the stability and existence of common Lyapunov functions for stable linear switching systems, Proceedings of the $36^{\text {th }}$ IEEE Conference on Decision and Control 2000, pp. 3723 -3724;

Shorten,R.N., Narendra,K.S., (1998b), Investigating the stability of a class of hybrid system, IEE Computing and Control Engineering Journal, 9(2), pp. 81-88, April, 1998;

Shorten,R.N., Narendra,K.S., (2000a), Necessary and sufficient conditions for the existence of a common quadratic Lyapunov function for a finite number of stable second order linear time-invariant systems, Proceedings of the 2000 American Control Conference, pp. 359-363;

Shorten, R., O'Cairbre, F. and Curran, P., (2000b), On the dynamic instability of a class of hybrid system, Proceedings of the IFAC 2000 Conference on Artificial Intelligence on Real Time Control, pp. 193-98;

Shorten, R. and O'Cairbre, F., (2002a), A new methodology for the stability analysis of pairwise triangularisable and related switching systems, Institute of Mathematics and its Applications, Journal of Applied Mathematics, In press;

Shorten, R. N., Curran, P., Wulff, K. and Mason, O. (2002b) Stability theory for switched linear systems, In preparation.

Wulff. K., Shorten, R. and Curran, P., (2002), On the 45degree region and the uniform asymptotic stability of second order parameter varying and switched systems, International Journal of Control, In Press;

Ye, H., A. N. Michel, and L. Hou, (1998), Stability Theory for Hybrid Dynamical Systems, IEEE Transactions on Automatic Control, 43(4);

\section{Appendix - Aerodynamic Modelling}

The aerodynamic torque, $\mathrm{T}_{\mathrm{rtr}}$, generated by the rotor depends nonlinearly on both the blade pitch angle, $\hat{p}$, rotor speed, $\Omega_{\mathrm{LS}}$, and the effective wind speed, $\mathrm{V}$; that is,

$$
\mathrm{T}_{\text {rtr }}=\mathrm{T}\left(\hat{\mathrm{p}}, \Omega_{\mathrm{LS}}, \mathrm{V}\right)
$$

For each effective wind speed, $\mathrm{V}$, above the rated wind speed, the rated aerodynamic torque, $\mathrm{T}_{\mathrm{o}}$, and rotor speed, $\Omega_{\mathrm{Lso}}$, are attained at a unique pitch angle, $\mathrm{p}_{\mathrm{v}}$. These pitch angles together with their corresponding effective wind speeds define the locus of equilibrium operating points.

In low wind speeds, the power generated by a wind turbine is limited by the available energy in the wind. At high wind speeds, the power generated is limited by the capacity of the generator. The boundary between these operating regimes, in steady operation, is the rated wind speed. This is defined as the minimum steady wind speed at which the turbine generates electricity at the capacity of the power-train (so, for the present machine, the minimum wind speed at which the power developed is $1 \mathrm{MW}$ ). Below this wind speed, the pitch angle of the rotor blades is held at zero degrees and the turbine is operated with the aim of maximising energy capture. Above the rated wind speed, the pitch angle of the rotor blades is adjusted to spill excess wind energy with the aim of maintaining energy capture at the rated level of $1 \mathrm{MW}$.

\section{Above Rated Wind Speed}

When operating above the rated wind speed the aerodynamic nonlinearity is separable (Leithead et al. 1999) in the sense that

$$
\mathrm{T}_{\mathrm{rtr}}\left(\hat{\mathrm{p}}, \Omega_{\mathrm{LS}}, \mathrm{V}\right)=\mathrm{g}(\mathrm{V})-\mathrm{h}\left(\hat{\mathrm{p}}, \Omega_{\mathrm{LS}}\right)
$$


for some nonlinear functions $\mathrm{h}$ and $\mathrm{g}$. The importance of this result is that it establishes that from a control viewpoint the wind turbine can be viewed as a nonlinear system with static nonlinearity $h$, subject to the external wind disturbance $\mathrm{g}(\mathrm{V})$. It is emphasised that the function $\mathrm{h}$ is independent of the wind speed. Consequently, the aerodynamics can be approximately linearised by suitably augmenting the plant using $\hat{\mathrm{h}}^{-1}, \hat{\mathrm{A}}_{\mathrm{u}}, \hat{\mathrm{A}}_{\mathrm{u}}{ }^{-1}$ which are suitable approximations to, respectively, $\mathrm{h}^{-1}$, the actuator dynamics and the inverse actuator dynamics. Exact linearisation is achieved when there is no approximation error. Assume that

1. The position saturation limit within the pitch actuator is neglected; that is, the controller is assumed to enforce this limit before the physical actuator limit are encountered.

2. $\hat{A}_{u}{ }^{-1}$ is selected such that $A_{u} \hat{A}_{u}{ }^{-1}$, essentially, has unity gain for the admissible signals, p. A requirement on the controller is to attenuate high frequency signals. Moreover, all physical actuators attenuate high frequency signals. Hence, the spectrum of $\mathrm{p}$ may be assumed to have little energy at high frequencies. Furthermore, it can be assumed that the actuator behaviour is reasonably well known over the range of admissible control signals; that is, for a linear actuator, its transfer function is known over the control bandwidth of the system. (In practice, straightforward laboratory or field tests can be employed to determine such actuator characteristics).

3. The mapping $\mathrm{h}\left(\hat{\mathrm{h}}^{-1}\left(\mathrm{~K}_{\mathrm{p}} \mathrm{p}, \Omega_{\mathrm{LS}}\right), \Omega_{\mathrm{LS}}\right)$ may be approximated by $\mathrm{K}_{\mathrm{p}} \Delta\left(\mathrm{p}, \Omega_{\mathrm{LS}}\right) \mathrm{p}$ for some suitable function $\Delta$ and constant gain $\mathrm{K}_{\mathrm{p}}$ (selected such that $\mathrm{p}$ and $\hat{\mathrm{p}}$ are comparable in magnitude).

4. The nonlinear wind disturbance function $\mathrm{g}(\mathrm{V})$ can be approximated by the quadratic mapping $\mathrm{K}_{\mathrm{V}} \mathrm{V}^{2}$ with appropriate gain, $\mathrm{K}_{\mathrm{V}}$.

Under these conditions, it follows that when the plant is suitably augmented then when operating above the rated wind speed the aerodynamics can be modelled as

$$
\mathrm{T}_{\mathrm{rtr}}\left(\mathrm{p}, \Omega_{\mathrm{LS}}, \mathrm{V}\right)=\mathrm{K}_{\mathrm{V}} \mathrm{V}^{2}-\mathrm{K}_{\mathrm{p}} \Delta\left(\mathrm{p}, \Omega_{\mathrm{LS}}\right) \mathrm{p}
$$

\section{Below Rated Wind Speed}

During operation below the rated wind speed, the pitch angle is held at zero degrees and therefore the aerodynamic torque is given by $\mathrm{T}\left(0, \Omega_{\mathrm{LS}}, \mathrm{V}\right)$. For the machine considered here, it can be shown that the dynamics are relatively insensitive to variations in rotor speed at below rated wind speeds. Employing a quadratic approximation to the wind speed dependence therefore leads to the below rated aerodynamic model (neglecting stall)

$$
\mathrm{T}\left(0, \Omega_{\mathrm{LS}}, \mathrm{V}\right)=\mathrm{K}_{\mathrm{V}} \mathrm{V}^{2}
$$

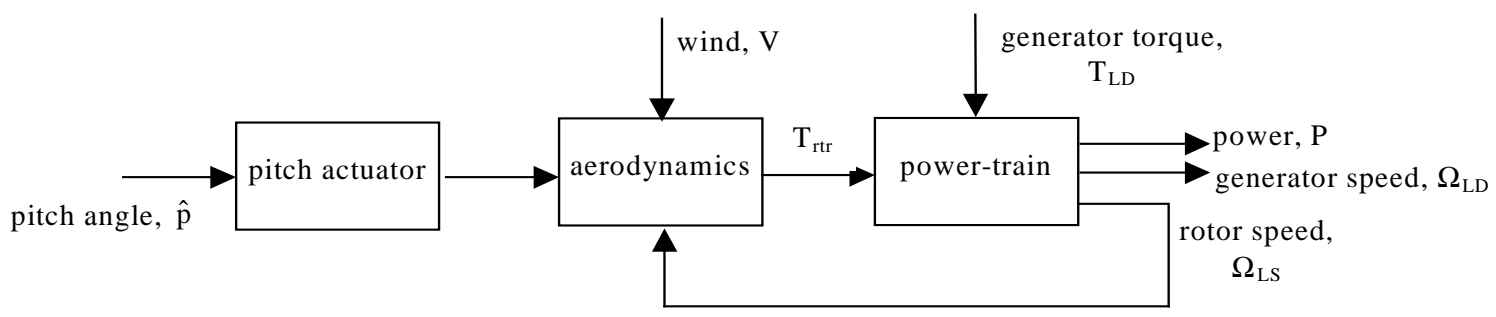

Figure 11 Block diagram representation of wind turbine 


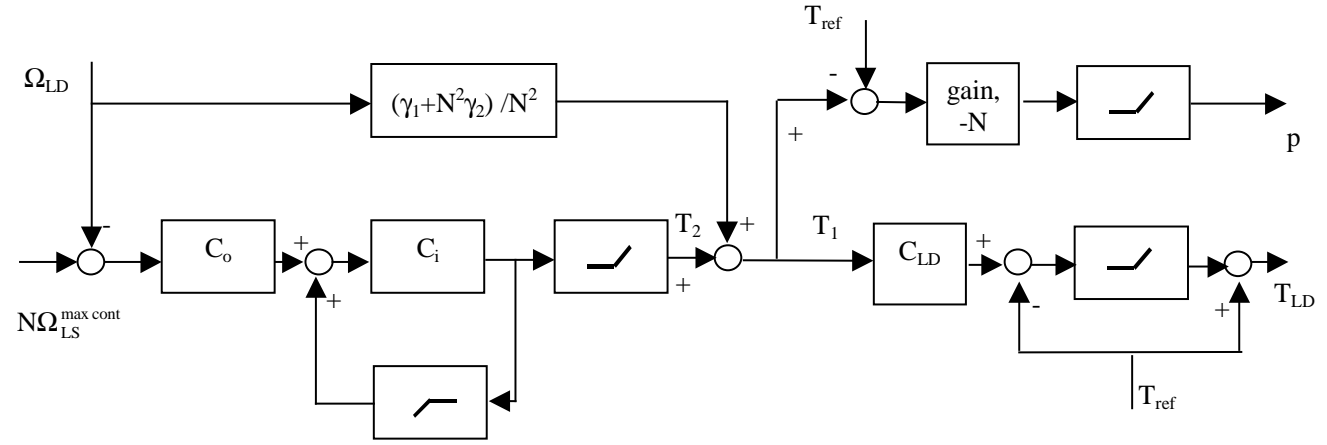

Key:

\begin{tabular}{l}
-1 denotes an element which only passes positive signals. \\
\hline$\leftarrow$ denotes an element which only passes negative signals.
\end{tabular}

Figure 12 Implementation of full-envelope controller

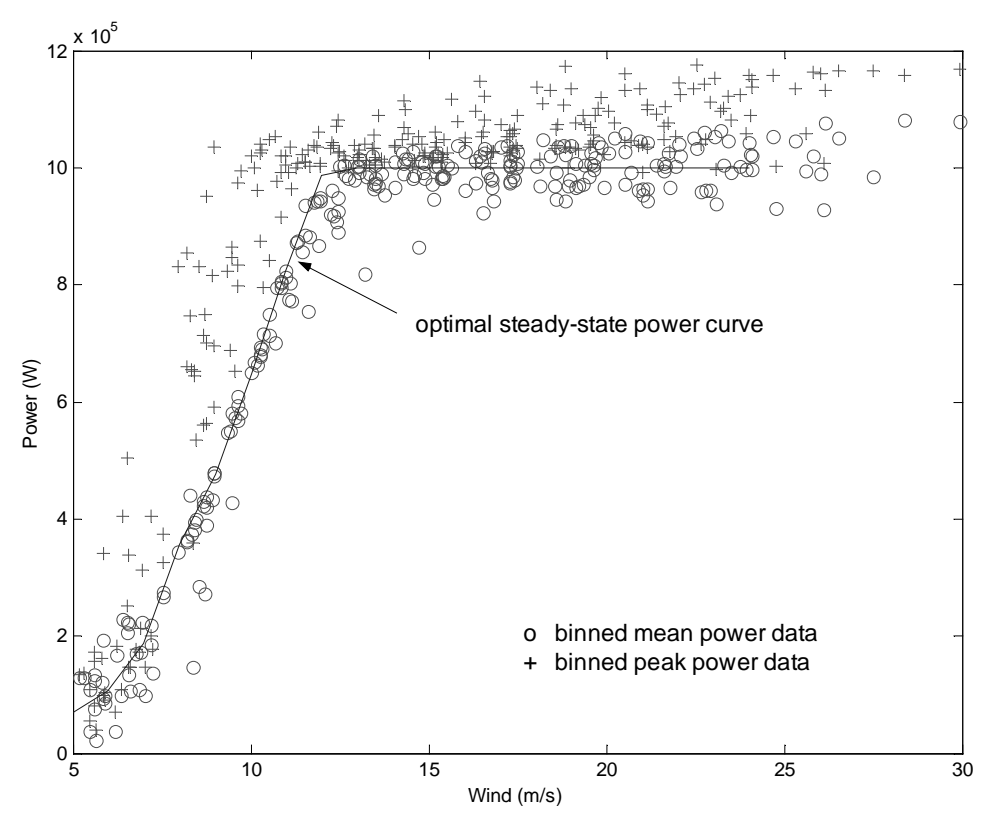

Figure 13a Performance plots for baseline controller. Measures of energy capture and fatigue loads. 


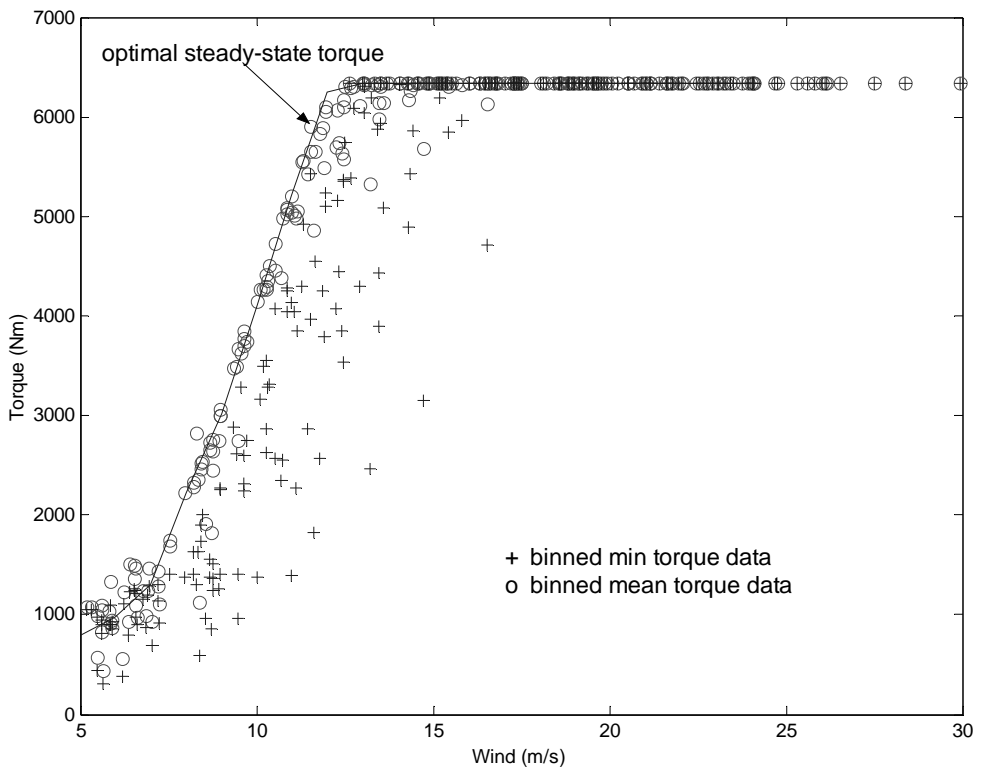

Figure 13b Performance plots for baseline controller. Measures of $T_{L D}$ control activity.

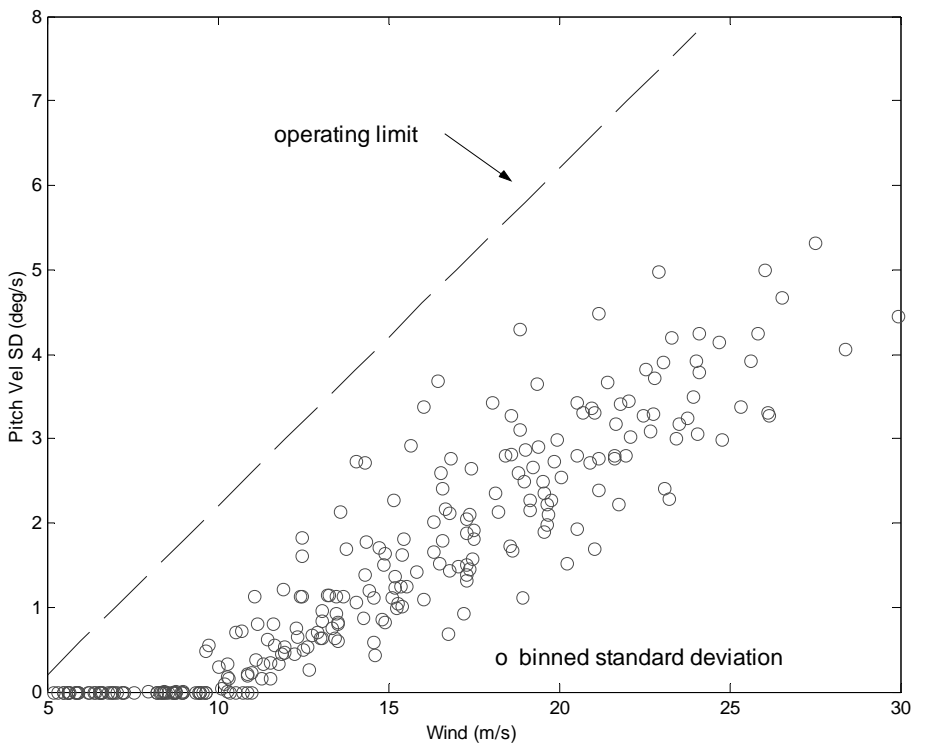

Figure 13c Performance plots for baseline controller. Pitch actuator activity. 


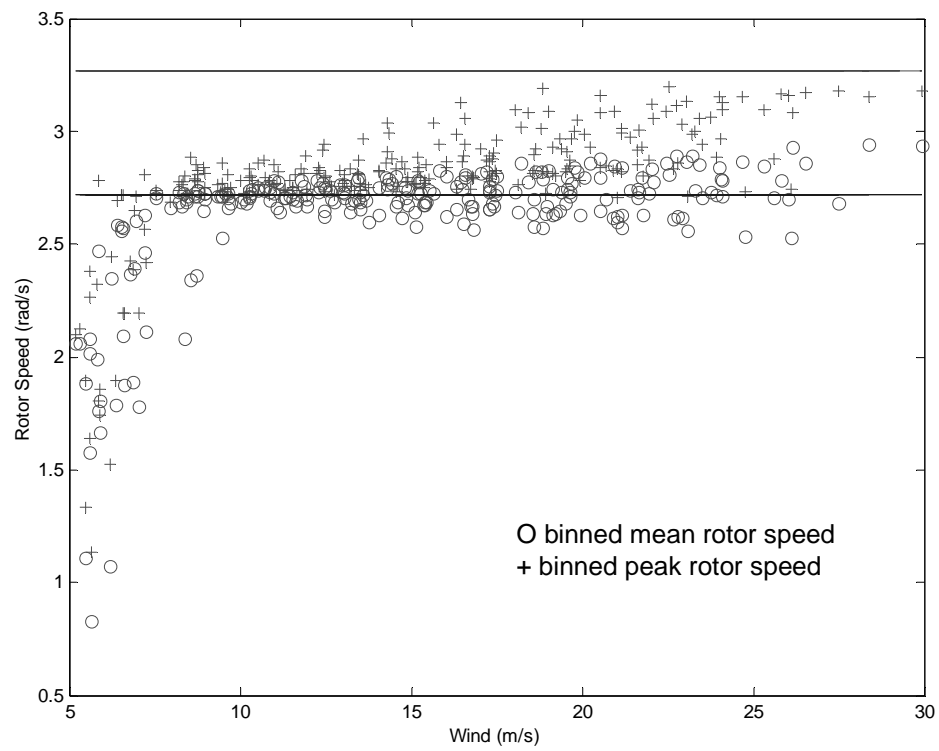

Figure 13d Performance plots for baseline controller. Rotor speed. 